\title{
The Small Angle Tile Calorimeter in the DELPHI Experiment
}

S.J. Alvsvaag ${ }^{1)}$, M. Bari ${ }^{12)}$, G. Barreira ${ }^{5)}$, A.C. Benvenuti ${ }^{2)}$, M. Bigi ${ }^{11)}$, M. Bonesini ${ }^{7)}$, M. Bozzo ${ }^{4)}$, T. Camporesi $^{3)}$, H. Carling ${ }^{6)}$, V. Cassio ${ }^{11)}$, L. Castellani ${ }^{9)}$, R. Cereseto ${ }^{4)}$, F. Chignoli ${ }^{7)}$, G. Della Ricca ${ }^{12)}$, D.R. Dharmasiri ${ }^{8)}$, M.C. Espirito Santo ${ }^{5)}$, E. Falk ${ }^{6}$, A. Fenyuk ${ }^{10)}$, P. Ferrari ${ }^{7)}$, D. Gamba ${ }^{11)}$, V. Giordano ${ }^{2)}$, Yu. Gouz ${ }^{10)}$, M. Guerzoni $^{2}$, S. Gumenyuk ${ }^{7)}$, V. Hedberg ${ }^{6)}$, G. Jarlskog ${ }^{6)}$, A. Karyukhin ${ }^{10)}$, A. Klovning ${ }^{1)}$, A. Konoplyannikov ${ }^{10)}$, I. Kronkvist ${ }^{6)}$, L. Lanceri ${ }^{12)}$, R. Leoni ${ }^{7)}$, O.A. Maeland ${ }^{1)}$, A. Maio ${ }^{5)}$, R. Mazza $^{7)}$, E. Migliore ${ }^{11)}$, F.L. Navarria ${ }^{2)}$, P. Negri ${ }^{7)}$, B. Nossum ${ }^{8)}$, V. Obraztsov ${ }^{10)}$, A. Onofre ${ }^{5)}$, M. Paganoni ${ }^{7)}$, M. Pegoraro $^{9)}$, L. Peralta ${ }^{5)}$, L. Petrovykh ${ }^{7)}$, M. Pimenta ${ }^{5)}$, P. Poropat ${ }^{12)}$, M. Prest ${ }^{12)}$, A.L. $\operatorname{Read}^{8)}$, A. Romero ${ }^{11)}$, N. Shalanda ${ }^{10)}$, L. Simonetti ${ }^{11)}$, T.B. Skaali ${ }^{8)}$, B. Stugu ${ }^{1)}$, F. Terranova ${ }^{7)}$, B. Tome ${ }^{5)}$, E. Torassa ${ }^{11)}$, P.P. Trapani ${ }^{11)}$, M.G. Verardi ${ }^{2)}$, E. Vallazza $^{12)}$, E. Vlasov $^{10)}$, A. Zaitsev ${ }^{10)}$

\begin{abstract}
The Small angle TIle Calorimeter (STIC) provides calorimetric coverage in the very forward region of the DELPHI experiment at the CERN LEP collider. The structure of the calorimeters, built with a so-called "shashlik" technique, gives a perfectly hermetic calorimeter and still allows for the insertion of tracking detectors within the sampling structure to measure the direction of the showering particle. A charged-particle veto system, composed of two scintillator layers, makes it possible to trigger on single photon events and provides e- $\gamma$ separation. Results are presented from the extensive studies of these detectors in the CERN testbeams prior to installation and of the detector performance at LEP.
\end{abstract}

(To be submitted to Nuclear Instruments and Methods A)

\footnotetext{
1) Department of Physics, University of Bergen, Bergen, Norway

2) Dipartimento di Fisica, Università di Bologna and INFN, Bologna, Italy

3) CERN, European Organization for Nuclear Research, Geneva, Switzerland

4) Dipartimento di Fisica, Università di Genova and INFN, Genoa, Italy

5) LIP, IST, FCUL, UCP, Lisbon and Coimbra, Portugal

6) Department of Physics, University of Lund, Lund, Sweden

7) Dipartimento di Fisica, Università di Milano and INFN, Milan, Italy

8) Department of Physics, University of Oslo, Oslo, Norway

9) Dipartimento di Fisica, Università di Padova and INFN, Padua, Italy

10) Institute for High Energy Physics, Serpukov, Russia

11) Dipartimento di Fisica, Università di Torino and INFN, Turin, Italy

12) Dipartimento di Fisica, Università di Trieste and INFN, Trieste, Italy
} 


\section{The Small Angle Tile Calorimeter in the DELPHI Experiment}

The Small angle TIle Calorimeter (STIC) provides calorimetric coverage in the very forward region of the DELPHI experiment at the CERN LEP collider. The structure of the calorimeters, built with a so-called "shashlik" technique, gives a perfectly hermetic calorimeter and still allows for the insertion of tracking detectors within the sampling structure to measure the direction of the showering particle. A charged-particle veto system, composed of two scintillator layers, makes it possible to trigger on single photon events and provides e- $\gamma$ separation. Results are presented from the extensive studies of these detectors in the CERN testbeams prior to installation and of the detector performance at LEP.

\section{INTRODUCTION}

At $\mathrm{e}^{+} \mathrm{e}^{-}$colliders the luminosity is monitored by measuring with high accuracy a well-known process, i.e., Bhabha scattering. The continuing progress in theoretical calculations and in Monte Carlo simulations of this process has reached an accuracy of $<1 \%$ which has stimulated the construction of better luminosity monitors for high precision $\mathrm{Z}^{0}$ physics at LEP. The difficulty in this task is given by the steep angular dependence of the Bhabha cross section $\left(d \sigma / d \theta \propto 1 / \theta^{3}\right)$ which makes it necessary to extend the measurement to as small angles as possible with a very well defined acceptance. In the DELPHI experiment $[1,2]$ an uncertainty of $1 \%$ in the luminosity measurement requires a control of the biases in the shower position at the inner edge of the acceptance at a level of $50 \mu \mathrm{m}$.

In the present phase of the LEP research program, at center-of-mass energies above the $\mathrm{W}^{+} \mathrm{W}^{-}$production threshold, one of the main goals is the search for the Higgs boson through the reaction $\mathrm{e}^{+} \mathrm{e}^{-} \rightarrow \mathrm{H}^{0} \mathrm{Z}^{0}$ with the $\mathrm{H}^{0}$ decaying into two jets and the $\mathrm{Z}^{0}$ into $\nu \bar{\nu}$. This process is the most sensitive to a massive $\mathrm{H}^{0}$ near the kinematic limit, but it requires a very hermetic detector in the forward region to eliminate background processes such as $\mathrm{e}^{+} \mathrm{e}^{-} \rightarrow \mathrm{Z}^{0} \gamma$ and $\mathrm{e}^{+} \mathrm{e}^{-} \rightarrow \mathrm{W}^{+} \mathrm{W}^{-} \rightarrow \mathrm{q} \overline{\mathrm{q}} \bar{\nu}$ with the photon or the electron escaping detection.

In 1992, the DELPHI Collaboration decided to meet these challenges by replacing the existing luminometer $[2,3]$ by a new calorimeter, the Small angle TIle Calorimeter (STIC) [4]. This detector has completed the calorimetric coverage in the forward region and provided a monitoring of the luminosity with an uncertainty of $0.9 \%$ when taking data on the $\mathrm{Z}^{0}$ peak.

The addition of a charged-particle veto system to the detector allows DELPHI to take advantage of the rather large cross-section for $\mathrm{e}^{+} \mathrm{e}^{-} \rightarrow \mathrm{Z}^{0} \gamma$ in order to measure the invisible decay width of the $\mathrm{Z}^{0}$. This measurement requires a trigger on single photons and thus relies on an efficient and fast photon identification system. Two layers of scintillators, read out with $10 \mathrm{~m}$ long optical fibers, provide such a system.

Off-energy electrons created in bremsstrahlung interactions between beam particles and residual gas molecules in the beampipe constitute the largest source of background to a single photon study. The insertion of two layers of silicon strip shower maximum detec- tors within the sampling structure makes it possible to measure the shower direction and thereby significantly reduce the off-energy electron background.

In the following the most salient features of the construction of the STIC, the veto system and the silicon strip shower maximum detectors will be described. This is followed by a presentation of the testbeam studies of these devices. Finally, the performance of the detectors at LEP will be discussed.

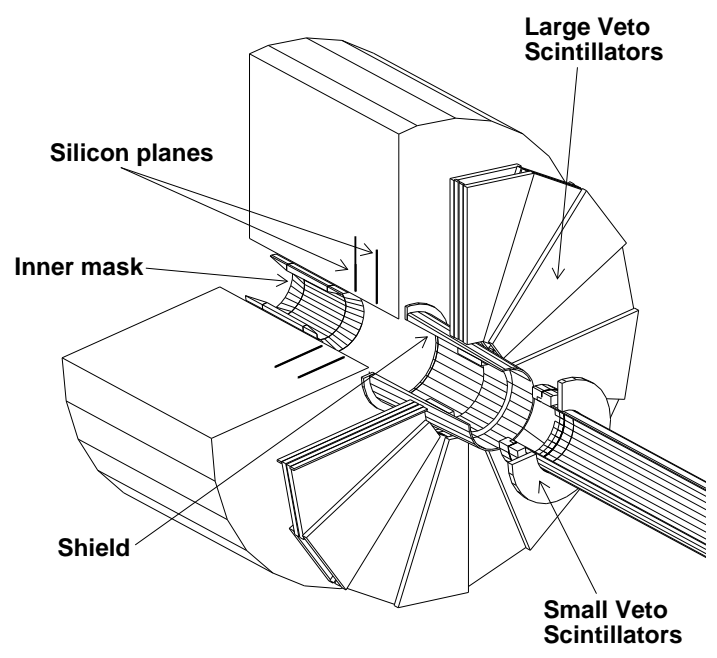

Figure 1. Drawing of one of the two calorimeters showing the silicon shower maximum detector and the veto scintillator counters. The "shield" and the "inner mask" are tungsten rings used to protect the central tracking chambers in DELPHI against low-energy photon background.

\section{THE CALORIMETER}

The STIC is a sampling lead-scintillator calorimeter where the blue light produced in the scintillator by an electromagnetic shower is carried to the photodetectors at the back of the calorimeter by means of plastic optical fibers ${ }^{1}$ doped with green wave length shifter (WLS). The novel feature of the calorimeter is given by a projective tower structure with continuous converter plates providing full hermeticity and eliminating non-uniformities in the energy response typical of calorimeters built with separate towers.

\footnotetext{
${ }^{1}$ This type of calorimeter has been nicknamed shashlik calorimeter from the Russian word for skewer since the fibers traversing the calorimeter layers look like skewers.
} 


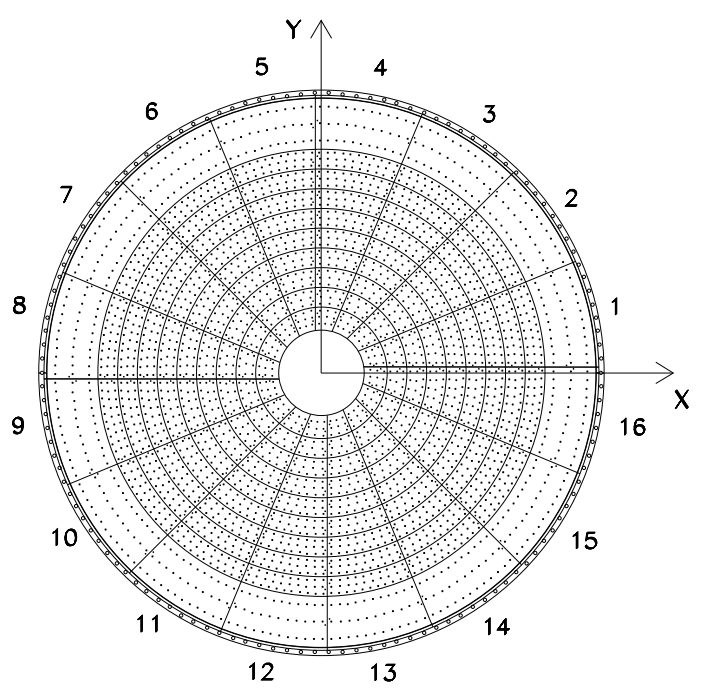

Figure 2. The first scintillator plane with the tower structure consisting of 16 sectors and 10 rings for a total of 160 towers for each calorimeter. The holes for the fibers are also shown.

The detector [5] consists of two cylindrical calorimeters placed on either side of the DELPHI interaction region at a distance of $2200 \mathrm{~mm}$, covering the angular region $29-185 \mathrm{mrad}(65-420 \mathrm{~mm} \text { in radius })^{2}$. Each calorimeter is made up of two half-cylinder modules mounted around the beampipe (Figure 1) and supported by the LEP low-beta quadrupole-girder. A STIC module consists of 47 layers of $3 \mathrm{~mm}$ thick lead plates and $3 \mathrm{~mm}$ thick scintillator tiles. Two layers of silicon strip detectors were inserted into the sampling structure after 7 and 13 sampling layers. The total depth of the calorimeter is $367.5 \mathrm{~mm}$ corresponding to 26 radiation lengths.

The fibers run perpendicularly to the lead (scintillator) layers through precision punched (drilled) holes and collect the light over the whole depth of the calorimeter. They are uniformly distributed over the calorimeter cross section (with about 0.79 fiber $/ \mathrm{cm}^{2}$ ), and the large fiber density, pioneered by Russian groups [6], reduces to a large extent the non-uniformities in light collection that are normally present with wavelength shifting techniques [7].

The scintillators have been cut into segments (tiles) in order to produce a tower structure with 10 rings and 16 sectors as shown in Figure 2. The radial width of the scintillator rings in the first layer is $35 \mathrm{~mm}$ for the inner ring $1,30 \mathrm{~mm}$ for rings 2 to 9 and $78 \mathrm{~mm}$ for the outer ring 10 . In order to prevent particles from tunneling through the fiber holes, the tower structure was made such that while all towers point to the interaction point

\footnotetext{
${ }^{2}$ In the DELPHI coordinate system, the $x$ axis points towards the center of LEP, the $y$ axis points upwards and the $z$ axis is in the direction of the electron beam. $\theta$ is the polar angle w.r.t. the $z$ axis and $\phi$ is the azimuthal angle around the $z$ axis. The radius $(R)$ from the beamline in STIC is given by $R(\mathrm{~mm})=2200 \cdot \tan (\theta)$.
}

in the radial direction, they are twisted by three degrees in azimuth. To obtain this, the border of the sectors were displaced by $375 \mu \mathrm{m}$ from one plane to the next, resulting in a total shift of $18 \mathrm{~mm}$ from the first to the last layer.

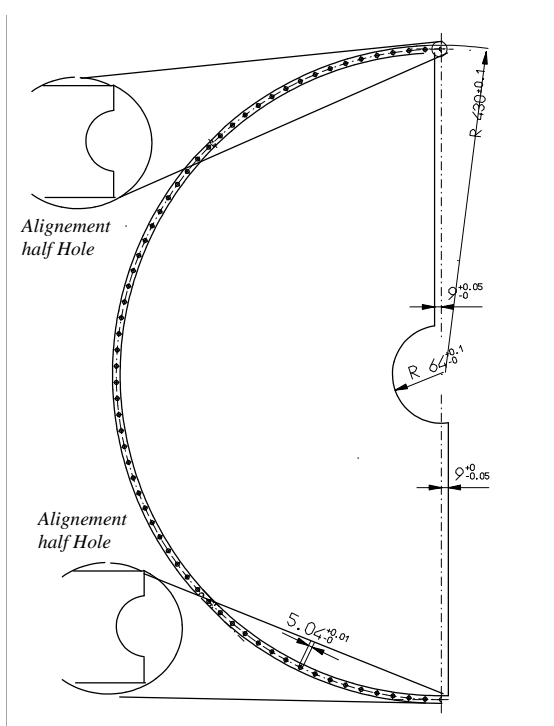

Figure 3. A drawing of one of the lead converter plates showing the aluminum ring around the edge.

\subsection{Mechanical construction}

The converter plates were made of lead plates strengthened by two steel foils (100 $\mu \mathrm{m}$ thick) glued to both sides of the lead using a resin pre-impregnated fiberglass cloth (resulting in a total thickness of $3.4 \mathrm{~mm}$ ). A schematic drawing of a converter plane is shown in Figure 3. Around the outer edge is an aluminum ring (7.5 mm wide) which acts as the support structure for one converter/scintillator layer. Aluminum was chosen as support material because its thermal expansion coefficient is very close to that of the lead-steel plates and this reduces the geometrical distorsions that could be caused by thermal stress. The radial thickness of the ring is minimal in order to reduce the inactive part of the calorimeter. A stack of 49 layers are held together by 62 steel rods $(\oslash 5 \mathrm{~mm}$ ) going through the holes in the ring. A half-hole (shown enlarged in Figure 3) at each end of the aluminum ring was used for positioning of the layer during the stacking and for guidance of the two half-cylinder modules when they were mounted around the beampipe.

The requirement of a very high mechanical accuracy motivated the following production technique:

- The scintillators were made of injection molded polystyrene doped with $1.5 \%$ paraterphenyl and $0.05 \%$ POPOP. A total of 470 different shapes of scintillator tiles were needed to complete the tower 
structure. The scintillator tiles were attached to the converter plates by means of precision pins $(\oslash 1 \mathrm{~mm})$ located in reference holes drilled in the scintillator and converter layers. The tiles were machined from a slab of scintillator in one operation (edges, fiber holes and positioning pin holes). The positions of the pin holes with respect to the edges were measured on an optical bench and the differences to the expected values were less than $30 \mu \mathrm{m}$ after sampling $10 \%$ of the tile production;

- The converter plates were then machined to the final shape and positioned on a numerically controlled punching machine which made all the fiber holes;

- The aluminum ring was glued to the edge of the converter plate. The plate was then positioned on a numerically controlled milling machine located in a room maintained at $23.5 \pm 0.5^{\circ} \mathrm{C}$, and the two reference half-holes on the aluminum ring and the holes for the alignment of the scintillator tiles were made. The distances between the centers of the two reference half-holes were the same to within $20 \mu \mathrm{m}$ for all layers (verified with an optical bench in a controlled temperature environment). The thicknesses of the aluminum rings were measured to be within $2 \mu \mathrm{m}$ of their nominal values. This translates into a difference in thickness between the various STIC modules of less than $100 \mu \mathrm{m}$;

- White $120 \mu \mathrm{m}$ thick Tyvek $^{\circledR}$ paper $^{3}$ was inserted between the absorber and the scintillator plane in order to increase the light collection, and also between tiles for optical separation. This paper was cut and the fiber and pin holes were made with a numerically controlled laser mill;

- The mounting of the scintillator tiles and the Tyvek ${ }^{\circledR}$ paper on the converter plate was made manually by using the two precision positioning pins in each tile.

The overall accuracy of the assembly was determined by measuring, on a high-precision $\mathrm{X}-\mathrm{Y}$ table equipped with optical measuring devices, the center and the radius of the circumferences defined by the edges of the first three tile rings (for all the calorimeter layers). The resulting distribution showed that the centers and the radii were less than $50 \mu \mathrm{m}$ from the specification in all layers.

\subsection{Optical fibers}

Two types of WLS fibers have been used in STIC: Kuraray Y7 with single cladding made of fluorinated plexiglass until 1995 and Kuraray Y11 with double cladding from 1996 onwards. The WLS fibers absorb light at $\sim 420 \mathrm{~nm}$ and have an emission peak at $\sim 500 \mathrm{~nm}$. The Y7 fibers had a length varying between 500 and $517 \mathrm{~mm}$, while the Y11 are $20 \mathrm{~mm}$ longer. The fibers were polished at both ends using an air-cushion diamond mill ${ }^{4}$.

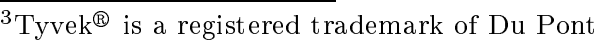

One end was aluminized by sputtering ${ }^{5}$ to increase the light collection.

The light yield and the attenuation length of all the fibers in the calorimeters were measured before assembly. For this purpose, an automatic test-bench based on a computer-controlled scanning table, with a precision of $20 \mu \mathrm{m}$, was used. In the test-bench a thin scintillator excited by a radioactive ${ }^{90} \mathrm{Sr}$ source was moved along the fiber, and the light output from the fibers was measured with a photomultiplier.

In order to avoid local light yield non-uniformities in the calorimeters, the measured light yield and the attenuation length were used to group the fibers. During the assembly, fibers from the same group were used for each individual tower. Approximately $10 \%$ of the 10,000 fibers studied were discarded, either because visual inspection revealed damage or because the measured attenuation length was less than $130 \mathrm{~cm}$ or because there were defects in the individual light attenuation pattern.

\begin{tabular}{|l|l|}
\hline & \\
Photocathode type & Bialkali \\
Useful photocathode diameter & $22 \mathrm{~mm}$ \\
No. of dynodes & 2 \\
Anode dark current & $\sim 0.1 \mathrm{nA}$ \\
Quantum eff. at $500 \mathrm{~nm}$ & $\sim 10 \%$ \\
Typical gain $(\mathrm{HV}=-900 \mathrm{~V}, \mathrm{~B}=0 \mathrm{~T})$ & $\sim 30$ \\
& \\
\hline
\end{tabular}

Table 1. Characteristics of the Hamamatsu R2149-03 tetrode.

\subsection{Phototetrodes and front-end electronics}

The light from the fibers coming from one tower was viewed through a $5 \mathrm{~mm}$ air gap by a 1" Hamamatsu R2149-03 phototetrode. The main characteristics of this device are given in Table 1 . The tetrode amplification chain consists of a bialkali photocathode, a finemesh dynode, a fine-mesh anode and a plate dynode. Secondary electrons from the fine-mesh dynode are collected by the anode, in part directly after emission and in part after multiplication on the plate dynode. The tetrodes were tested in a laboratory set-up with the operating voltages set to $-900 \mathrm{~V},-500 \mathrm{~V}$ and $-180 \mathrm{~V}$ for the cathode, the fine-mesh dynode and the plate dynode respectively [8]. The total gain was then about 30 due to a multiplication factor three between the cathode and the fine-mesh dynode and a multiplication factor ten between the fine-mesh dynode and the anode.

Inside the $1.2 \mathrm{~T}$ magnetic field of DELPHI, the tetrodes gain is reduced. The field affects the acceler-

\footnotetext{
${ }^{4}$ GEBEX, Uster, Switzerland.

${ }^{5}$ Precitrame, Tramelan, Switzerland.
} 
ated electrons inside the tetrode, producing a significant dependence of the relative gain on the orientation angle. The upper plot in Figure 4 shows the dependence of the gain on the angle, at a field of $1.2 \mathrm{~T}$, for five different tetrodes, while the bottom plot shows the average profile for a sample of 25 tetrodes. An increase in gain of $20 \%$ is obtained by tilting the tetrodes by $15^{\circ}$, and the tetrodes were therefore mounted with this angle in STIC. Figure 5 shows the ratio of the gain at $1.2 \mathrm{~T}$ to $0 \mathrm{~T}$ for a sample of 280 tetrodes when these were mounted at an angle of $15^{\circ}$ w.r.t. the magnetic field direction.
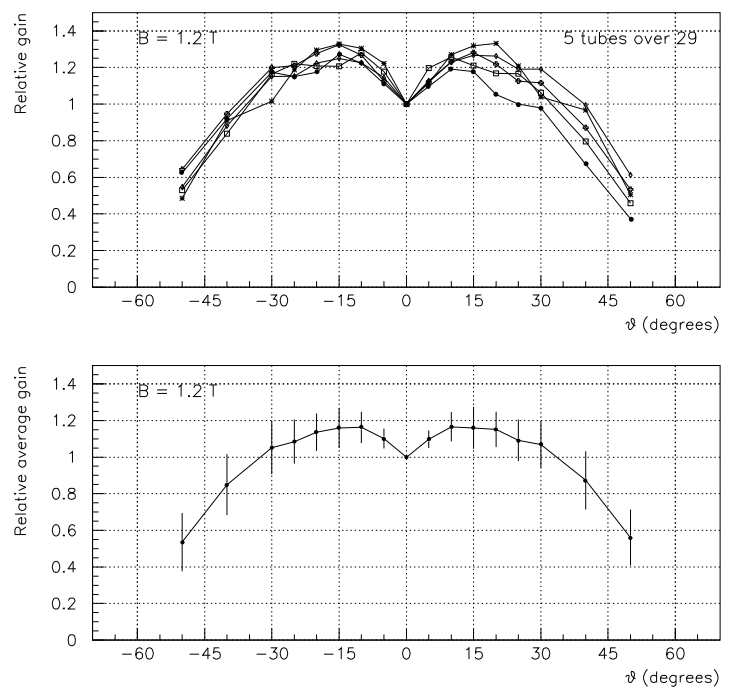

Figure 4. Distribution of gains as a function of the orientation angle with respect to the magnetic field direction for a sample of 25 R2149-03 Hamamatsu tetrodes in a $1.2 \mathrm{~T}$ field. The upper plot shows the distribution for individual tubes, while the bottom plot shows an average profile. The error bars give the rms spread.

Each tetrode was placed inside an aluminum housing, containing a charge sensitive JFET preamplifier and a high voltage divider. The preamplifier incorporates a high-pass filter to eliminate the microphonics noise induced by mechanical vibrations of the dynode structure. The photodetector contribution to electronic noise comes from the anode capacitance $(\leq 10 \mathrm{pF})$ and from the dark current $(\sim 0.1 \mathrm{nA})$. The equivalent noise charge, using a Gaussian shaping time of 500 ns, was 250 electrons. The preamplifier gain was adjusted by means of a resistor to $0.24 \mu \mathrm{V} /$ electron with a maximum output signal of $1.5 \mathrm{~V}$. The average rise time was $15 \mathrm{~ns}$, followed by an exponential fall $(\tau \sim 10 \mu s)$ to match the shaper input characteristics. The differential output signals are fed via $40 \mathrm{~m}$ long cables to the counting room where they are digitized. For testing purposes, a set of programmable pulse generators allows injection of a precise charge signal at the input of each preamplifier.

\subsection{The LED monitoring system}

A luminosity measurement with $1 \%$ o systematic uncertainty requires a monitoring of all detector channels

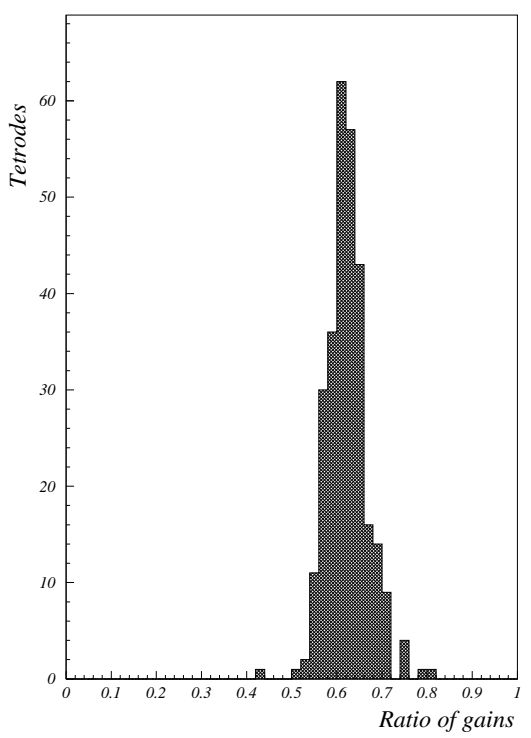

Figure 5. Distribution of the ratio of gains at $1.2 \mathrm{~T}$ to $0 \mathrm{~T}$ for a sample of 280 R2149-03 Hamamatsu tetrodes.

and of the trigger system. A LED-based monitoring system was built for this purpose [9].

Four driver boards were mounted on the front face of each of the two calorimeters. Each board generates the electrical pulses for flashing up to 40 light emitting diodes. The $\operatorname{LEDs}^{6}$ are inserted into plexiglass tiles which are arranged in a layer in front of the first converter plane of the calorimeter in a way similar to that of the regular STIC scintillator planes. The blue light from the LED propagates inside the tile and is absorbed by the fibers which continue out of the calorimeter into holes in the tiles. The re-emitted green light propagates inside the fibers to the photocathode of the tetrode. This design allows monitoring of the stability of the whole light collection chain.

The driver board can deliver pulses with independently selected amplitudes to any combination of up to 40 LEDs and the selection is made via an 8 bit control bus. The temperature stability of the system is better than $0.3 \%$ o per degree and the rms variation of the charge injected into a LED is $<1 \%$ o for the maximum signal. The stability of the LED signals was studied during a seven hour time period, i.e., a typical LEP fill lifetime. The variations of the signals, measured with the calorimeter readout chain, was within $\pm 1.5 \%$.

The number of photoelectrons produced in the tetrodes was measured with the LED system as a function of the high voltage and the amplitude of the LED signal. The effective number of photoelectrons $\left(N_{p e}^{e f f}\right)$ can be calculated from $\sigma_{p e} / E=1 / \sqrt{E \cdot N_{p e}^{e f f}}$ where $\sigma_{p e} / E$ is the energy resolution measured by the LED system. $N_{p e}^{e f f}$ was found to be independent of the amplitude but showed a dependence on the high voltage with a maximum around $200-250 \mathrm{~V}$ and a decrease at higher voltages (Figure 6). At LEP, the detector was

\footnotetext{
$\overline{{ }^{6} 102 \mathrm{CR}-\mathrm{ND}, \text { manufactured by CREE }}$
} 
operated at $550 \mathrm{~V}$, which gave $N_{p e}^{e f f}=306$ per GeV averaged over all the phototetrodes $(\mathrm{rms}=64)$ in a magnetic field of $1.2 \mathrm{~T}$. Without magnetic field this number was $30 \%$ higher.

The number of photoelectrons produced at the photocathode $\left(N_{p e}\right)$ can be calculated from $N_{p e}^{e f f}$ if the dynode amplification is taken into account since $N_{p e}=$ $\left(1 / \delta_{1}+1 /\left(\delta_{1} \cdot \delta_{2}\right)\right) \cdot N_{p e}^{e f f}$ where $\delta_{i}$ is the amplification of dynode $i$ [10]. In the laboratory set-up at $-900 \mathrm{~V}$, $N_{p e}$ was estimated to be a factor 1.64 (1.37) larger than $N_{p e}^{e f f}$ at $1.2 \mathrm{~T}(0 \mathrm{~T})$.

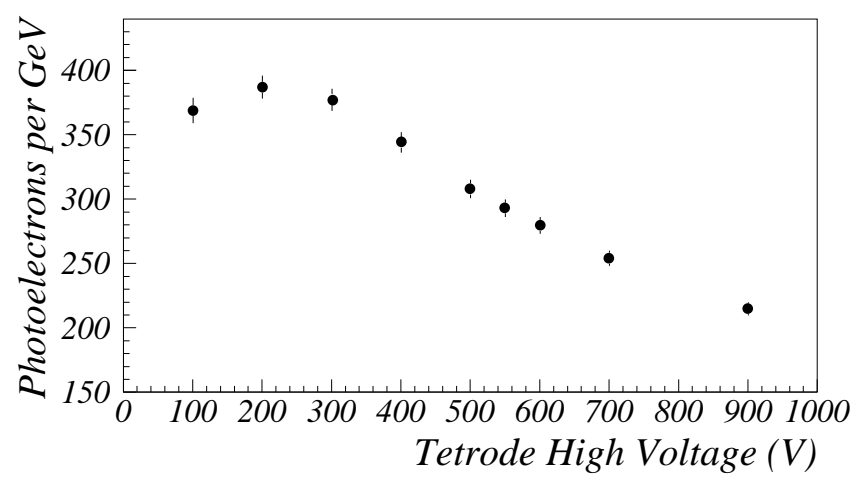

Figure 6 . The effective number of photoelectrons per $\mathrm{GeV}$ as a function of tetrode high voltage. $N_{p e}^{e f f}$ was measured as an average over all phototetrodes in the calorimeter, and the measurement was done with a magnetic field of $1.2 \mathrm{~T}$.

\section{CHARGED-PARTICLE VETO SYSTEM}

Photons are identified and triggered on by requiring that there is no signal in two sets of scintillators called the large and the small veto counter. An unusual feature of these counters is that the light produced in the scintillators is transported out of the DELPHI magnetic field to the photomultipliers by WLS fibers and clear fiber optical cables.

\subsection{The large veto counters}

The STIC large veto counters [11] consist of 64 trapezoidal scintillators assembled into two matching planes on each side of DELPHI, at a distance of between 2010 and $2050 \mathrm{~mm}$ from the interaction point (Figure 1). The center of the inner edge of the scintillators is at a radius of $86 \mathrm{~mm}$ and the outer edge is at a radius of $379 \mathrm{~mm}$.

The counters are made of $10 \mathrm{~mm}$ thick Bicron BC-408 plastic scintillator. The light is collected from each scintillator by $2 \times 855 \mathrm{~cm}$ long Kuraray Y7 WLS fibers glued with optical cement ${ }^{7}$ in two $8.35 \mathrm{~mm}$ wide and $1.2 \mathrm{~mm}$ deep grooves, machined along each of the two longest edges of the scintillator. The inner and outer edges of the counter are coated with a reflector paint made of titanium dioxide in a water soluble binder ${ }^{8}$. The scintillator is wrapped with Tyvek $^{\circledR}$ paper to improve light collection and it is made light-tight with black plastic

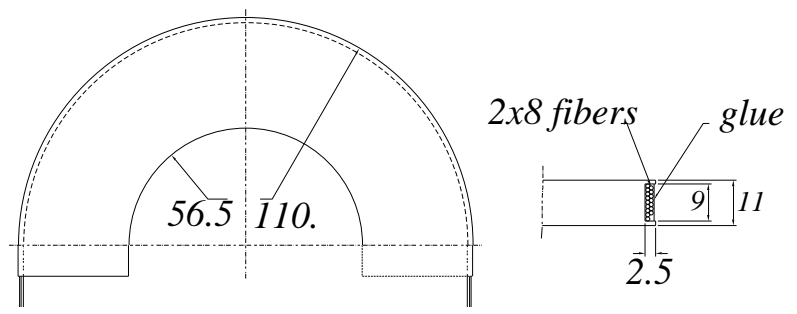

Figure 7. A drawing of one of the four small veto scintillators and the groove along the outer edge which contains the WLS fibers. All distancies are in $\mathrm{mm}$.

material.

The light is read out by a 10 stage Hamamatsu H3165 photomultiplier located outside the DELPHI magnetic field. The photomultiplier is coupled to the WLS fibers via a $10 \mathrm{~m}$ long fiber optic cable made of 16 clear $\mathrm{Ku}-$ raray polystyrene fibers $(\oslash 1 \mathrm{~mm})$. The photomultiplier side of the cable was terminated by gluing the 16 fibers to the inside of a plexiglass cylinder and polishing the surface with a diamond mill. At the counter side, the fiber optic cable was split into two bundles of eight fibers, and these bundles were glued into optical connectors. The WLS fibers from the scintillator were terminated with matching connectors. The light transmission across the connector was found to vary between 85 and $90 \%$.

The counters were tested with cosmic rays using a $1.2 \mathrm{~m}$ long clear fiber cable and one H3165 photomultiplier. The average response of the counters was found to be 32 photoelectrons per minimum ionizing particle (MIP). The clear fiber cables, tested with a green light emitting diode coupled to each of the connectors, gave a distribution of the light transmission with an rms spread of $9 \%$.

\subsection{The small veto counters}

During data taking at the $\mathrm{Z}^{0}$ peak, the inner edge of the STIC acceptance was defined by a tungsten ring (see Section 8). However, at higher energies this ring was removed. This increased the coverage of DELPHI, but at the same time it created a gap in the STIC veto coverage in the critical region close to the beampipe.

In order to close this gap, scintillator counters were installed directly on the DELPHI beampipe, using a flange for support (Figure 1). These two so-called small veto counters are located on either side of the interaction point at a distance of $1800 \mathrm{~mm}$ and each counter consists of two scintillators. These are $10 \mathrm{~mm}$ thick and have a half-ring shape with an inner radius of $56.5 \mathrm{~mm}$, an outer radius of $110 \mathrm{~mm}$ and a $15 \mathrm{~mm}$ straight section at each end (Figure 7). The size of the inner radius is dictated by the size of the beampipe, $55 \mathrm{~mm}$ in radius, while the outer radius was chosen to match approximately the transition region between the STIC tower rings 2 and 3 .

\footnotetext{
${ }^{7}$ Bicron BC-600.

${ }^{8}$ Bicron BC-620.
} 
The light is collected by 16 WLS fibers, $1 \mathrm{~mm}$ in diameter and $110 \mathrm{~mm}$ long, with one end polished and the other aluminized by sputtering. The fibers are divided into two bundles which, except for the length, are identical to those used in the large veto system. The bundles are glued with optical cement in two layers to the inside of a $9 \mathrm{~mm}$ wide and $1.5 \mathrm{~mm}$ deep groove machined on the outer perimeter of the counters, as shown in Figure 7. The first layer contains nine fibers, and the seven remaining fibers form a second layer in such a way as to optimize the light collection. The bundle with the fibers glued directly to the scintillator collects $\sim 60 \%$ of the light.

The counters were tested with cosmic rays before installation and gave a response of $\sim 12$ photoelectrons per MIP at the end of a $10 \mathrm{~m}$ long fiber optic cable.

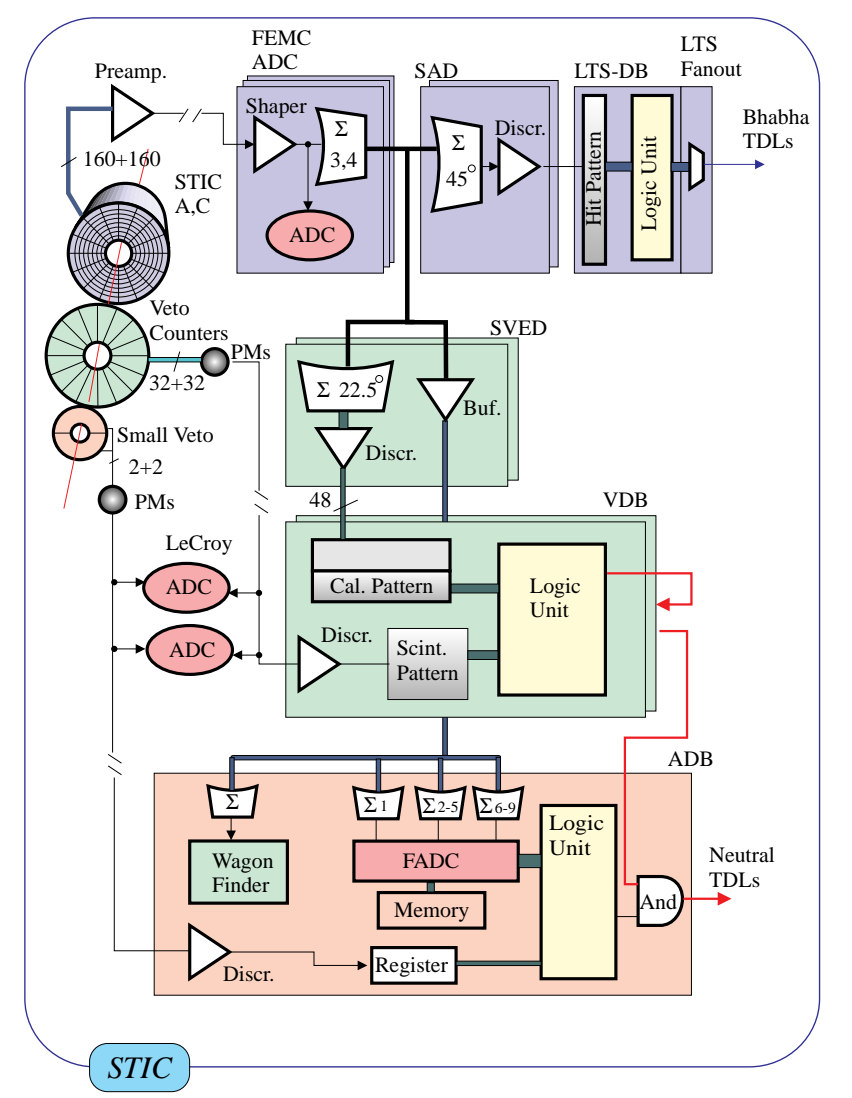

Figure 8. Block diagram of the trigger and the readout system.

\section{TRIGGER SYSTEM AND READOUT}

The trigger and the readout chain of the calorimeter and veto system are shown in Figure 8. The 320 signals from the preamplifiers of the calorimeters are sent to Fastbus shaper \& ADC boards developed originally for the forward lead glass calorimeter in DELPHI [12]. Analog sums of three or four towers are combined by another module (SAD) into $45^{\circ}$ overlapping sectors and the resulting signals are discriminated with two different thresholds. The low-threshold hit patterns are processed by a programmable logic unit (LTS-DB) [13] to create back-to-back Bhabha triggers used in the offline calculation of the luminosity. The high-threshold bits are used as a more selective trigger of Bhabha events for the online luminosity measurement.

The scintillator veto counters are used to trigger on single photon events. In this so-called neutral trigger, the calorimeter signals are combined in $22.5^{\circ}$ sectors and the resulting analog sums are discriminated (SVED) [14]. The signal from the 64 large veto counters are split and sent both to Fastbus $\mathrm{ADCs}^{9}$ and to discriminators (VDB) [15]. The hit patterns from the calorimeter and the large veto counters are combined in a logic unit to form the neutral trigger. The trigger allows a signal in at most one of the two scintillator planes in front of the shower. Both the veto counter sector directly in front of the shower and its two neighbours are considered, since the edges of the scintillator sectors overlap.

The four signals from the small veto counters are also split to a Fastbus ADC and to a set of discriminators (ADB). If any of these discriminators are set the neutral trigger is inhibited.

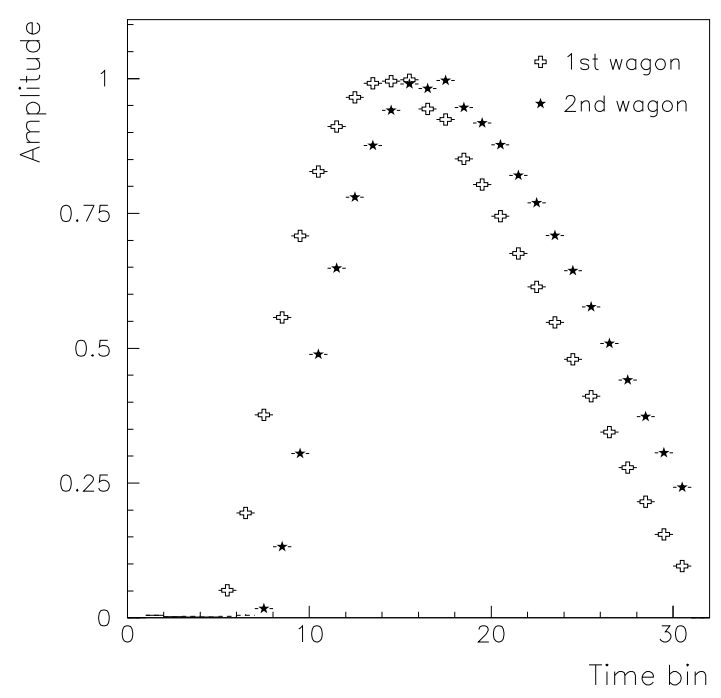

Figure 9. The pulse shape from two different showers produced by different wagons as measured by the FADC. Each bin corresponds to 140 ns.

In 1995, the LEP operation mode changed from colliding beams of eight bunches separated by $11 \mu$ s to collisions of four so-called bunchtrains each having up to 4 "wagons" or "minibunches" separated by 247 ns [16]. This entailed a loss of synchronization with the collisions at the level of $\pm 370 \mathrm{~ns}$ and a deterioration of the STIC

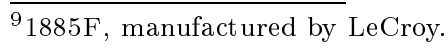




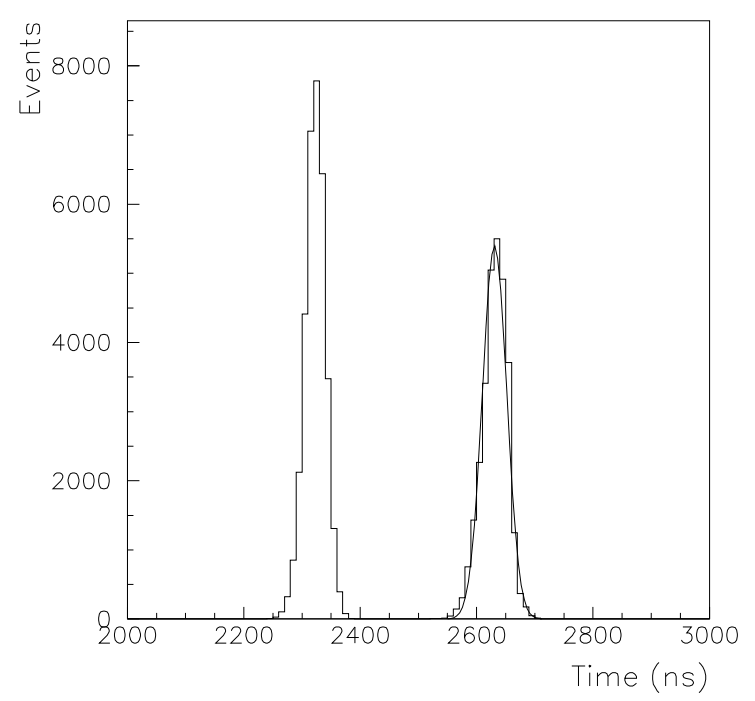

Figure 10. The time distribution of calorimeter signals determined from the pulse shape. The Gaussian fit to the second peak has $\sigma=21.0$ ns.

energy measurement. The STIC readout was modified to deal with the new situation. By using four sets of discriminators for the veto counter signals (each gated at the arrival time of a different wagon) it was possible to determine which wagon had caused the interaction. The veto counter hit pattern was also used to produce the gate for the calorimeter ADCs. During the periods when LEP was running in bunch train mode, the level of inefficiency (for Bhabha events) of the wagon tagging was negligible, and only $1.7 \%$ of the Bhabha online wagon tags were ambiguous. Furthermore, in most of these cases, it was possible to determine offline the true wagon number by comparison of the recorded hodoscope hit patterns with the position of the shower in the calorimeter and thus to correct the measured energy. The fraction of Bhabha events in which the offline wagon determination failed was about $0.3 \%$.

The veto counter cannot be used to determine the wagon in single photon events since they are selected on the basis of not having any signals in these detectors. Instead the calorimeter signal itself is digitized 32 times by 8-bits FADCs (ADB) with a sampling interval of $140 \mathrm{~ns}$ (Figure 9). From the position of the rising edge of the digitized pulse it was possible to determine the wagon number offline and to correct the energy measurement accordingly. Figure 10 shows the time distribution of calorimeter signals determined from such a pulse shape measurement. A clear separation between electrons from two wagons can be seen and the time resolution obtained with this method is $21 \mathrm{~ns}$.

The FADC values are also used to calculate the radial position of the calorimeter showers in the trigger logic. If the radius is less than $11 \mathrm{~cm}$ the neutral trigger is rejected. In this way, a large part of the off-energy electron background is suppressed and the trigger rate is greatly reduced.

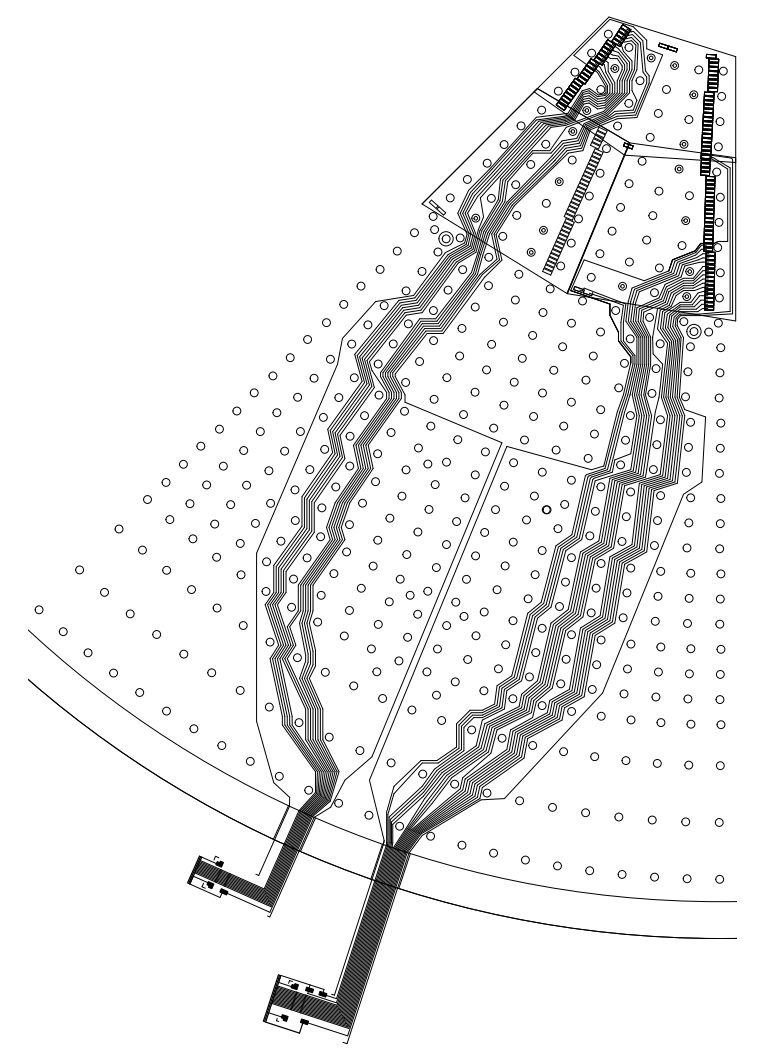

Figure 11. Layout of a $45^{\circ}$ sector of the silicon shower maximum detector with two of its eight Kapton cables shown. The strip structure is not drawn; only the connections to the strips are indicated. Note the holes for the calorimeter fibers which the signal wires have to avoid.

\section{THE SILICON STRIP DETECTOR}

Each STIC calorimeter was equipped with two planes of silicon shower maximum detectors [17]. The reasons for this were that

- it provides a means to reconstruct the shower axis with $\sim 10$ mrad accuracy and this improves the rejection of off-energy electron background;

- it improves the coordinate resolution and the twoshower separation;

- it provides a cross-check in the determination of the STIC calorimeter acceptance in the luminosity measurement.

The silicon planes were installed at depths of 4.0 and 7.4 radiation lengths inside the calorimeter and they cover the angular region between 32.5 and $79 \mathrm{mrad}$. Radially, the covered region starts at $71.5 \mathrm{~mm}(73.0 \mathrm{~mm})$ from the beam axis and extends to $174.2 \mathrm{~mm}(178.3 \mathrm{~mm})$ for the first (second) plane. The two planes are slightly different in order to match the projective geometry of the STIC calorimeter. Each plane consists of $1 \mathrm{~mm}$ thick ceramic tiles attached to a $3.5 \mathrm{~mm}$ thick aluminum plate and is aligned by three dowels. The silicon detector was attached, with conductive glue, to a metallized area of 

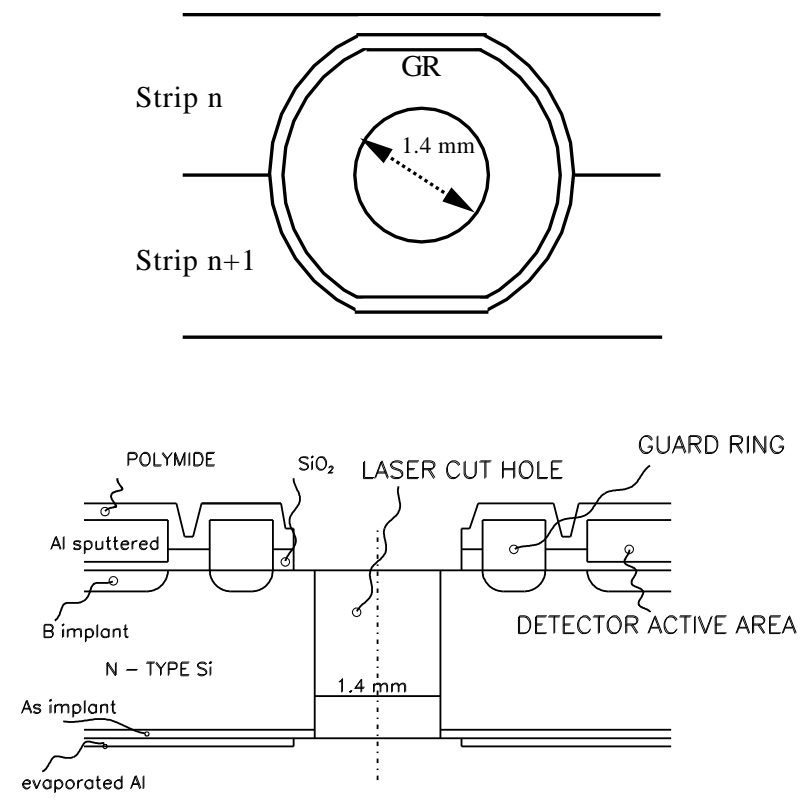

NOT TO SCALE

Figure 12. Layout of the region surrounding a fiber hole. Both a top and a side view are shown.

the ceramic which was used to provide the backplane biasing voltage for the diodes.

The detector is made of $300 \mu \mathrm{m}$ thick, high-resistivity n-type silicon, with p-type strips implanted on the front and an $\mathrm{n}^{+}$layer on the back. The circular strips cover $22.5^{\circ}$ in azimuthal angle, i.e., one calorimeter sector, and have a radial pitch of $1.712 \mathrm{~mm}(1.754 \mathrm{~mm})$ for the first (second) plane. The radial granularity was chosen, following simulation studies [18], to obtain an optimum spatial resolution. In both planes the silicon detectors are arranged in two concentric crowns. Each $45^{\circ}$ sector consists of three silicon wafers with one inner wafer covering two calorimeter sectors and two outer wafers each covering one calorimeter sector. The inner wafers have $2 \times 24$ radial strips while the outer wafers have 36 strips (Figure 11).

The main challenge in the detector construction was to make the $1.4 \mathrm{~mm}$ diameter holes in the silicon for the passage of the WLS fibers. Three different techniques for making the holes were tested: laser cutting, ultrasonic grinding and chemical etching. All three techniques gave satisfactory mechanical results. However, the best quality to price performance was obtained using laser cutting ${ }^{10}$. The accuracy obtained in the hole diameter was less than $10 \mu \mathrm{m}$. The layout of the region surrounding the holes is shown in Figure 12. The implanted strip is protected by a guard region extending to $300 \mu \mathrm{m}$ from the edge of the hole. The total passive zone around a hole has a diameter of about $3 \mathrm{~mm}$. The holes are always located between two strips, in order to have strip continuity.

In order to evaluate possible damage to the substrate, the strips with holes were compared to those without

${ }^{10}$ MICRON Semiconductor Ltd., UK. holes [19]. Figure 13 shows that there was a slight difference in capacitance, due to geometrical effects, between the two sets of strips while the full depletion voltage was the same. A special production of wafers without holes showed leakage currents comparable to those of the detectors with holes.
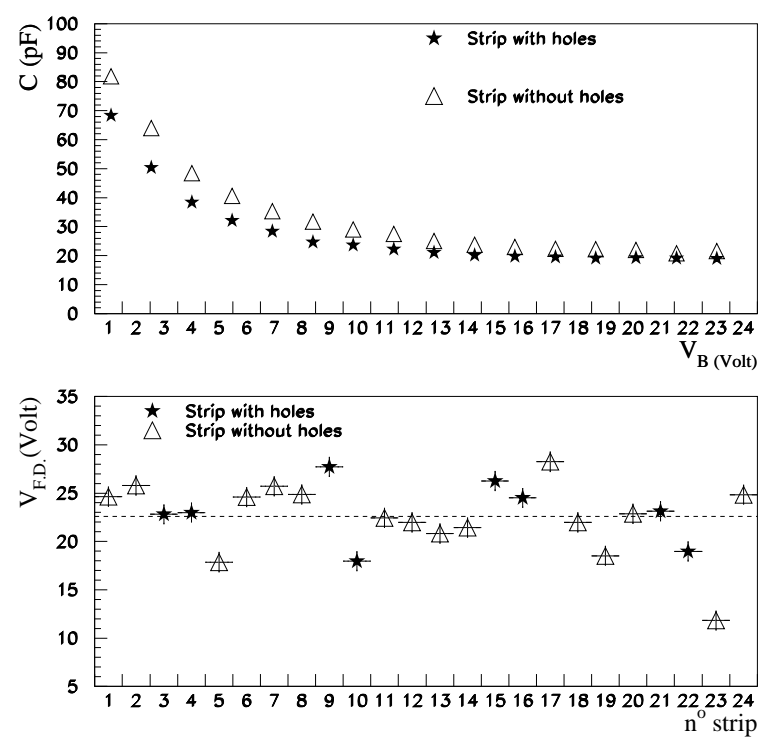

Figure 13. A comparison of the capacitances (top) and of the full depletion voltages (bottom) between strips with and without holes.

The strips were $\mathrm{AC}$ coupled to the readout and biased by means of a FOXFET scheme [20]. Each strip acts as an independent source, while the gate and the drain are common. The drain was put at the same voltage as the preamplifier $(5 \mathrm{~V})$ in order to avoid current flow in case of damage to the decoupling capacitance. A voltage between $30 \mathrm{~V}$ and $50 \mathrm{~V}$ (depending on the detector) was applied to the backplane, in order to achieve full depletion and to compensate for the voltage drop between the strip and the drain. An aluminum pad was used for charge collection on each strip. Two aluminum wires, $33 \mu \mathrm{m}$ in diameter, were used to bond each pad to a copper track ${ }^{11}$ deposited on a flexible cable which brings the signals to the outer edge of the calorimeter (Figure 11). The cable was made of a $50 \mu \mathrm{m}$ Kapton $^{12}$ substrate, $35 \mu \mathrm{m}$ copper tracks and a $50 \mu \mathrm{m}$ protective coating.

Each $45^{\circ}$ sector of each silicon plane is connected to a Microplex 4 (MX4) charge amplifier with 128 channels and multiplexed output, designed by the Rutherford Appleton Laboratory (UK) [21]. The signals from the strips are sampled and stored twice, once before the LEP beam crossing and once after all the charge in the silicon has been collected, and the difference between the two measurements is sent to a differential amplifier. The technique of double sampling provides a suppres-

\footnotetext{
${ }^{11}$ Mipot, Cormons, Italy.

${ }^{12}$ Manufactured by Du Pont.
} 
STIC SILICON FRONTEND ELECTRONICS

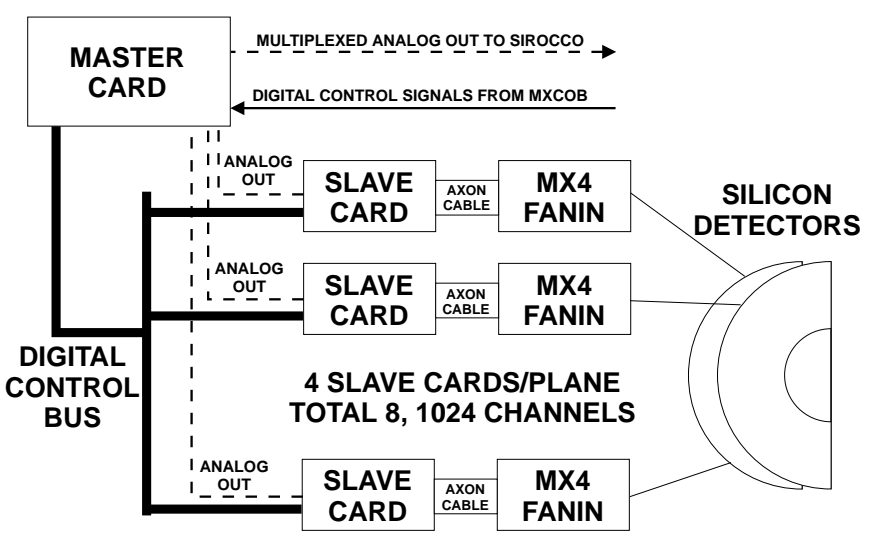

Figure 14. Scheme of the front-end electronics of the silicon detectors for a half cylinder STIC module. The silicon detectors are connected to the MX4 fanin card by flexible Kapton printed circuit cables. The fanins receive the digital control signal from and send the analog differential outputs to the slave cards which amplify the signals. The slave cards are plugged as daughterboards on a PCB on the front of the calorimeter. This same PCB is used to bus the digital and analog signals to a master card, which multiplexes 8-to-1 the analog signals before they are sent to the Fastbus SIROCCO in the counting room for digitization.

sion of common mode spurious signals induced at the detector level.

A special hybrid fanin card $^{13}$ matches the $0.5 \mathrm{~mm}$ track pitch on the Kapton cables to the $44 \mu \mathrm{m}$ pitch of the MX4 chip. It consists of a ceramic substrate on which a $1 \mu \mathrm{m}$ aluminum layer has been evaporated under vacuum and then imprinted by chemical etching. The connections between the Kapton cables and the tracks on the fanin were made by connectors ${ }^{14}$ soldered onto the fanin. Given the difficulties of soldering directly on the aluminum tracks, the solder pads were made by evaporating in sequence a layer of titanium $(0.05 \mu \mathrm{m})$, palladium $(0.15 \mu \mathrm{m})$ and gold $(0.30 \mu \mathrm{m})$, after which another layer of gold $(3 \mu \mathrm{m})$ was added by galvanization. At first only chromium and gold were used, but this led to unstable contacts to $10-15 \%$ of the pads.

A block diagram of the full front-end electronics chain is shown in Figure 14. The output signals from the amplifiers (slave cards) are sent to the control room via an 8-to-1 analog multiplexer (master card). The data from each detector are clocked, at a $1 \mathrm{MHz}$ frequency, into a Sirocco IV Fastbus module [22], where they are digitized by a flash ADC.

\footnotetext{
13 Designed at CERN and manufactured by Mipot, Cormons, Italy.

${ }^{14} \mathrm{ELCO}^{\circledR}$, series $6200-6201$.
}

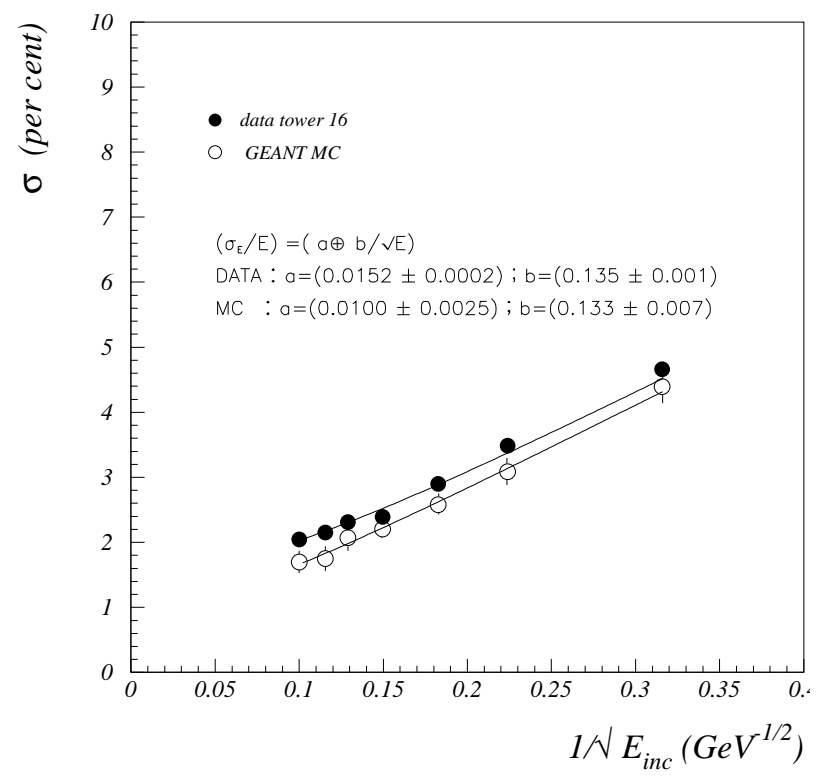

Figure 15. The measured and GEANT simulated energy resolution for electrons from a testbeam going into the center of one tower.

\section{PERFORMANCE IN A TESTBEAM}

\subsection{Studies of the calorimeter}

The conceptual design of the STIC detector was studied with a small prototype in 1992, to ascertain the energy resolution, the response uniformity and the accuracy of the position measurement [23]. These measurements were repeated with two different STIC modules in 1993 and the results can be summarized as follows:

- the energy resolution was $\sigma / E(\%)=(1.52 \pm 0.02) \oplus(13.5 \pm 0.1) / \sqrt{E} ;$

- the contribution to the resolution from electronic noise was negligible $(\sigma \simeq 90 \mathrm{MeV})$;

- the energy linearity was within $\pm 1 \%$;

- the energy deposited by muons was $4.5 \sigma$ above the pedestal.

Figure 15 shows a comparison of the measured energy resolution with the results of a GEANT [24] based Monte Carlo simulation [25]. In this simulation photons and electrons in the electromagnetic showers were tracked down to cut-off energies of $50 \mathrm{KeV}$ and $500 \mathrm{KeV}$, for photons and electrons respectively. The modulation of the energy response due to the presence of the fibers and the leakage of light between neighbouring scintillators was implemented in the GEANT Monte Carlo by means of light collection maps. These give, as a function of the position where the energy is deposited, the fraction of light collected in the same tower and in the neighbouring ones. The light collection maps were computed in a separate fast Monte Carlo program [26] that was tuned to reproduce the test-bench measurements of the fibers and the scintillators [27]. 


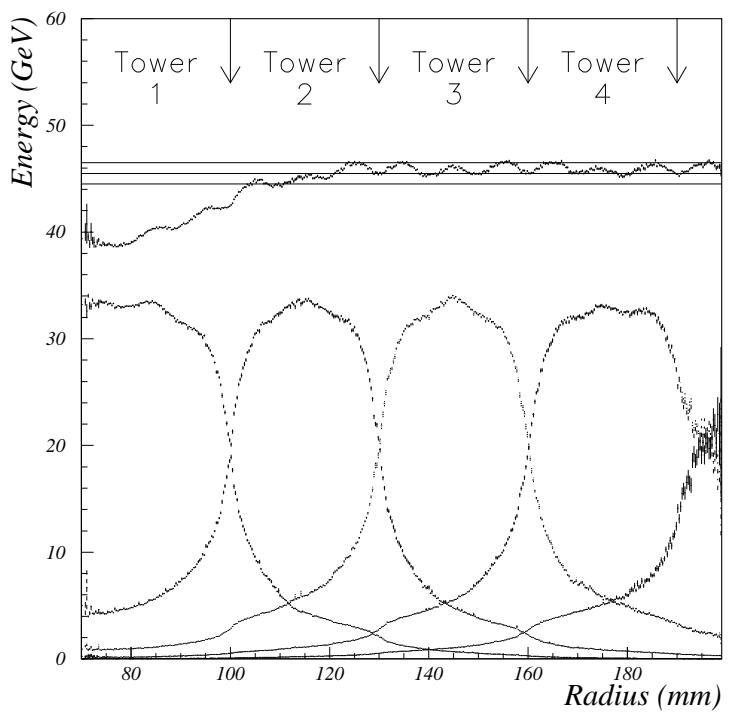

Figure 16. Spatial uniformity of STIC response. The curves in the lower half of the figure correspond to the energy deposited in each tower while the curve in the upper half gives the overall energy deposit. The lines indicate the $\pm 2 \%$ interval around the beam energy.

The comparison of the energy resolution formula obtained from the testbeam data and the GEANT simulation shows good agreement between the sampling terms while there is a difference in the constant term. This can most likely be attributed to the difficulties in simulating the light collection mechanism.

The spatial uniformity of the energy response was mapped by scanning the calorimeter radially with an electron beam. The impact point of the particle was measured with an accuracy of $<40 \mu \mathrm{m}$ using an external silicon microstrip telescope. The energy collected by each tower and the overall energy measured by the calorimeter are shown in Figure 16 as a function of the radial impact point. Above tower ring 1, where the energy measurement is not affected by the shower leakage, the response is modulated within a band of $\pm 2 \%$. This is due to the increase in light collection when the particle enters the calorimeter close to a fiber. The importance of the continuous absorber used in STIC is apparent in the uniformity of the response as the beam crosses the tower boundaries.

The radial position of a shower was obtained from the energy sharing between rings of calorimeter towers [28]. Radial scans were made of all tower rings and Figure 17 shows one such scan of tower rings 1 and 2 performed with a $45 \mathrm{GeV}$ electron beam and the external silicon microstrip telescope. In this measurement, the ratio of the energy deposited in the two rings is given as a function of the impact point from the telescope. The distance $d_{k}$ to the border between rings $k$ and $k+1$ was parametrized as a function of the estimator

$$
\epsilon=\ln \frac{\sum_{i=1}^{k} E_{i}}{\sum_{i=k+1}^{N_{\text {rings }}} E_{i}}
$$

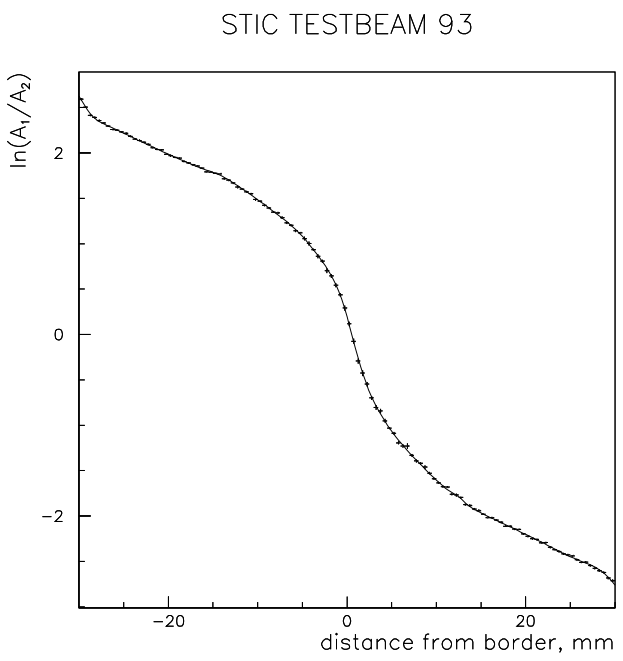

Figure 17. The ratio of the energy measured in tower ring 1 and tower ring 2 versus the impact point measured by the microstrip telescope.

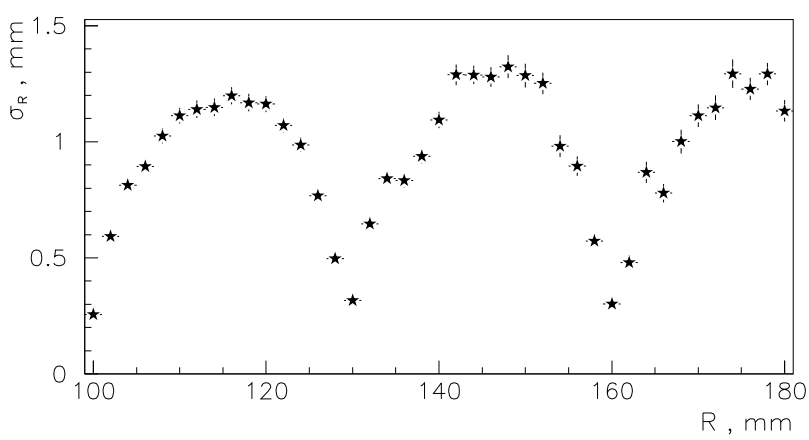

Figure 18. The radial resolution of the calorimeter versus radius.

where $E_{i}$ is the measured energy in ring $i$. The quantity $d_{k}(\epsilon)$ was measured for each border $k$ in the testbeam.

For each shower the distances to the outer and the inner borders of the ring with the maximum energy deposit were calculated. These two measurements were combined to improve the resolution near the center of the rings. Figure 18 shows the measured radial resolution as a function of the radius. It varies from $0.25 \mathrm{~mm}$ in a narrow region $( \pm 2 \mathrm{~mm})$ around the ring borders to $1.2-1.3 \mathrm{~mm}$ in the central regions of the rings.

In the same way as for the radial position, the algorithm reconstructing the azimuthal angle of the shower was based on the measured sharing of shower energy at the border between adjacent calorimeter sectors. The linear distance from the impact point to a border between two sectors was calculated with an estimator similar to (1). Since the towers are larger in the azimuthal direction than in the radial direction the azimuthal resolution is also poorer. The resolution in the azimuthal direction in, for example, ring 4 varies between $\sim 1 \mathrm{~mm}$ at the tower sector border and $\sim 4 \mathrm{~mm}$ at the tower center. 

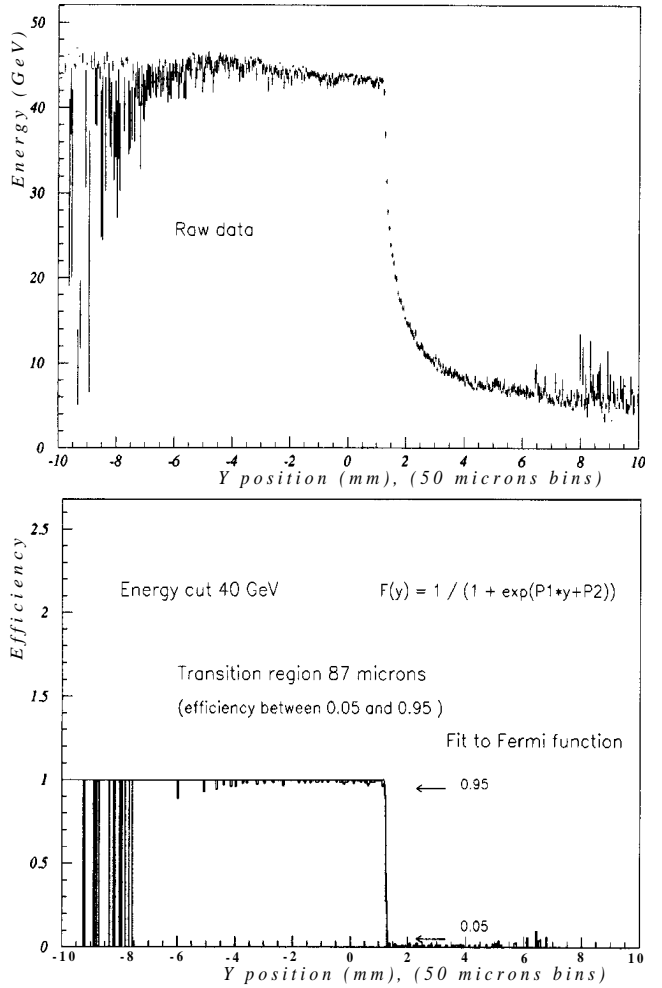

Figure 19. Energy deposited in the calorimeter as a function of the radial impact position $(y)$ (top). The average energy measured by the calorimeter (normalized to $45 \mathrm{GeV}$ ) as a function of the radial impact position (bottom). The region $y \geq 1 \mathrm{~mm}$ was covered by a tungsten mask. A fit of a Fermi function to the transition region at the mask border is superimposed.

In a luminosity monitor it is essential to have a good definition of the inner edge of the acceptance. This can be achieved either directly by measuring the energy sharing as described above or by using an accurately machined tungsten mask which translates a cut in energy into a very precise radial measurement [3,23]. The mask method was studied with the prototype calorimeter and a silicon microstrip telescope by scanning the edge of a $6 \mathrm{~cm}$ thick piece of tungsten [29]. Using an optimized value for the energy cut (e.g. $40 \mathrm{GeV}$ for $45 \mathrm{GeV}$ electrons), the transition region was measured. The transition region was defined as the region where the fraction of events with a shower energy larger than the energy cut goes from $5 \%$ to $95 \%$. The width of this region gives the sharpness of the inner edge of the used acceptance. The measurement, shown in Figure 19, gives a transition region of $87 \mu \mathrm{m}$ for a fit of a Fermi function and $82 \mu \mathrm{m}$ for a fit of a step function convoluted with a Gaussian distribution. The latter fit gives $\sigma=25 \mu \mathrm{m}$ which is close to the expected resolution of the measurement of the impact point. Therefore, it was concluded that the mask method defines the inner edge of the acceptance to better than $25 \mu \mathrm{m}$.

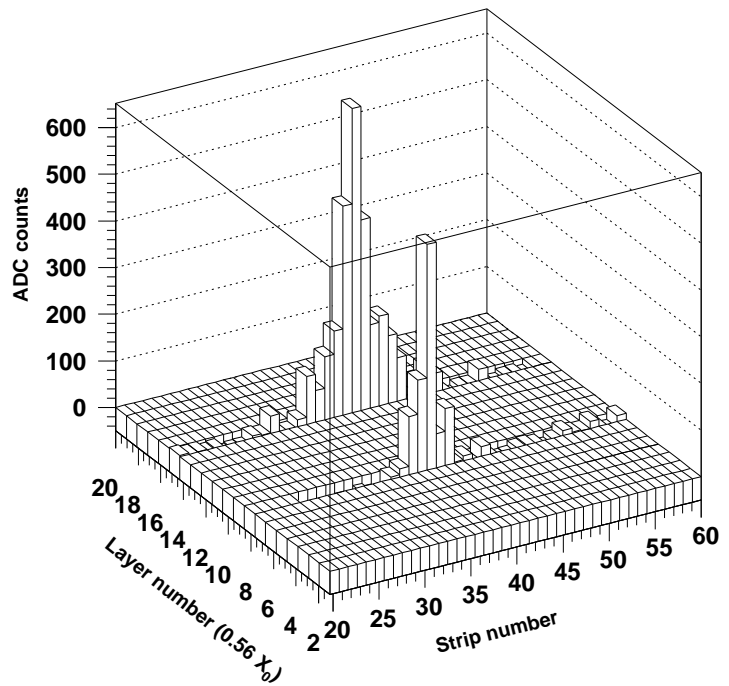

Figure 20. The online pulse height distribution for different strips after pedestal and noise subtraction (for one $45 \mathrm{GeV}$ testbeam electron).

\subsection{The silicon shower maximum detectors}

In 1994, a STIC calorimeter module equipped with silicon shower maximum detectors was exposed to a $45 \mathrm{GeV}$ electron testbeam. Both the silicon detectors and the readout chain were the same as those used in DELPHI.

The data showed the presence of a coherent noise source that produced a common shift of the baseline. Pedestal runs were taken between the testbeam runs to monitor the noise and to determine correlation coefficients between the various strips. The noise was then subtracted on an event-by-event basis by using the signals from the strips outside a shower and the correlation coefficients.

The measured signal-to-noise ratio in the strip with the maximum energy deposit was about 40 for a $45 \mathrm{GeV}$ electromagnetic shower. Figure 20 shows an example of an online pulse height distribution after pedestal and coherent noise subtraction.

As shown in Figure 21, the transverse profiles of the shower, measured by the two silicon planes, agree well with those of a simulation based on the GEANT program.

The radial position of the incoming particle was estimated, in both silicon planes, by means of a barycenter method applied to the five strips around the shower maximum, without correcting for possible strip-to-strip variations in gain. The distribution of the differences between the radial positions reconstructed by the two silicon planes (Figure 22) provides a measurement of the angular resolution in the reconstruction of the direction of the showering particle. The $0.63 \mathrm{~mm}$ FWHM of the distribution translates into an angular resolution of 13 mrad, since the distance between the two silicon planes is $51.7 \mathrm{~mm}$. This resolution is in good agreement with the $10 \mathrm{mrad}$ resolution aimed for in the proposal [4] for the detector. 

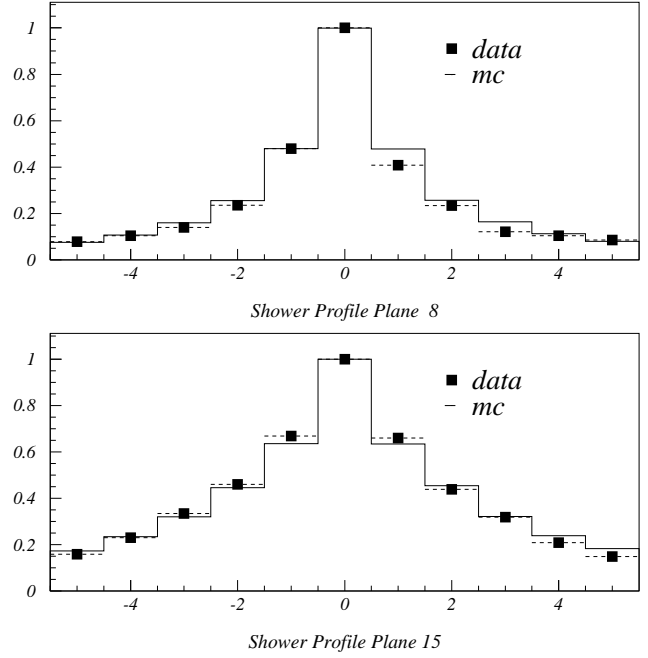

Figure 21. Transverse shower profiles, for $45 \mathrm{GeV}$ electrons, in the first (at $4.0 X_{0}$ ) and second (at 7.4 $X_{0}$ ) silicon plane. The testbeam data and the results of a GEANT simulation are compared.

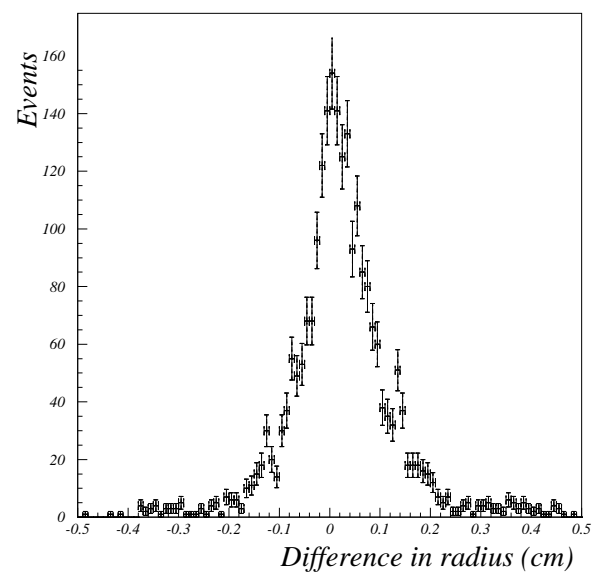

Figure 22. Difference between the radial positions reconstructed in the two silicon planes.

\subsection{The veto counters}

Two scintillator counters, covering one calorimeter sector, were mounted on a STIC module in a testbeam. They were positioned at approximately the same distance from the calorimeter as in DELPHI and were read out with the same type of photomultipliers and a $10 \mathrm{~m}$ long clear fiber cable. The purpose of the test was to measure the response of the counters to minimum ionizing particles (MIPs) and more importantly to determine the loss of photon initiated showers due to shower backsplash from the calorimeter, the so-called "albedo" effect.

The response of the veto counters, measured with $45 \mathrm{GeV}$ electrons, was independent of the position of the impact point and was $\sim 20$ photoelectrons per MIP. With a MIP defined by a cut on the pulse height at $3 \sigma$ above the pedestal, more than $99.7 \%$ of the electrons fulfilled the MIP requirement in both counters.

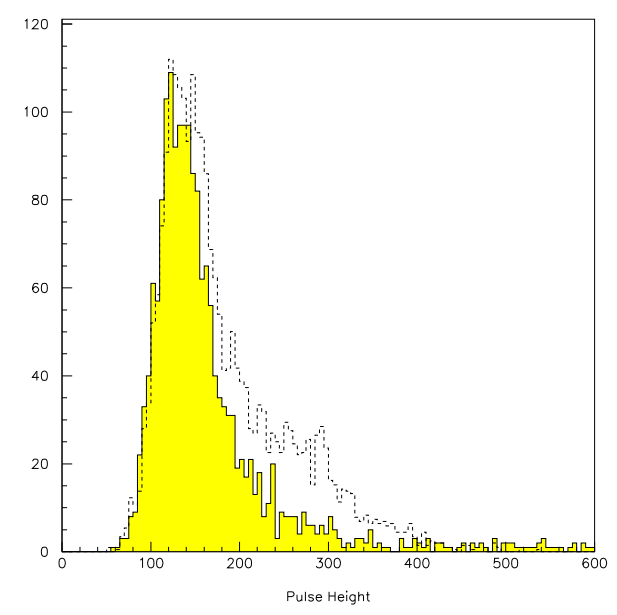

Figure 23. Pulse height distributions for electrons (the dashed histogram) and for pions (the shaded area).

The pulse height distribution for pions was quite different from that of electrons (Figure 23). The distribution for the electrons had a tail at large pulse heights, absent for the pions, and due to the albedo. When the counters were placed at a distance of $2 \mathrm{~m}$ in front of the calorimeter, the energy distributions for electrons and for pions were identical.

The most direct way to measure the albedo would be to expose the calorimeter and the veto counters to a photon beam and count the fraction of showers in STIC giving a pulse height in the veto counters consistent with that of a MIP. No photon beam was available, however, and therefore the pulse height from the counters was measured with the electron beam hitting the center of a calorimeter sector adjacent to the one covered by the scintillators or at a distance of one and half sectors away. The albedo was then defined to be the fraction of events identified as MIPs when the electron beam hits STIC but not the veto counters.

The results from a radial scan of two STIC sectors with a $45 \mathrm{GeV}$ electron beam are shown in Figure 24 as a function of the STIC tower ring. The figure shows the albedo for each counter separately and after requiring that both counters give pulse heights consistent with a MIP. In all cases the albedo was independent of the radial position of the impact point. For a single counter the albedo was $\sim 12 \%$ when the beam hit the center of the adjacent sector and $\sim 7 \%$ when the impact point was one sector further away. When both counters had to satisfy the MIP cut the albedo was reduced to $\sim 5 \%$ and $\leq 2 \%$ respectively.

The energy dependence of the albedo was measured with the beam going into the center of tower ring 5 in the sector next to the scintillators and by varying the energy of the beam. The data showed an increase of the albedo from $\sim 2 \%$ at $5 \mathrm{GeV}$ to $\sim 6 \%$ at $100 \mathrm{GeV}$ when the presence of a signal in both counters was used to define the albedo. 

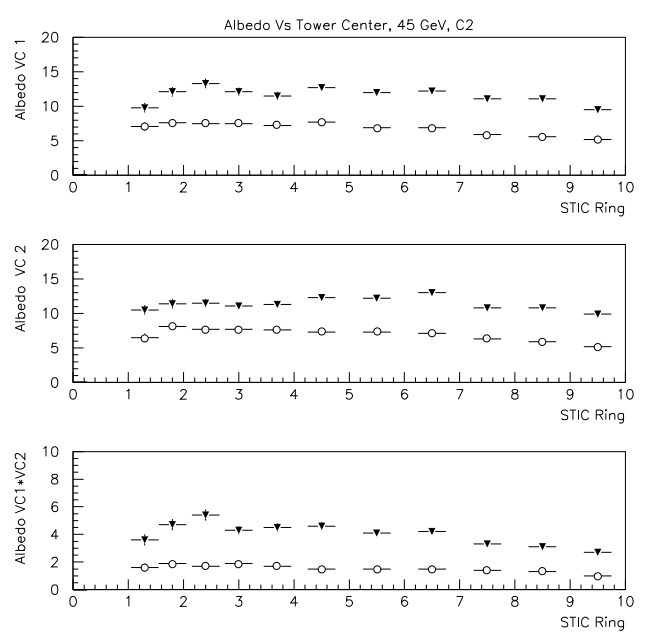

Figure 24. Fraction of events with a pulse height consistent with a MIP when the beam hits a calorimeter sector adjacent to the veto counters (triangles) and when the impact point is one sector further away (circles). The two upper plots show the albedo for each counter separately, and the lower plot is for the coincidence between the two counters.

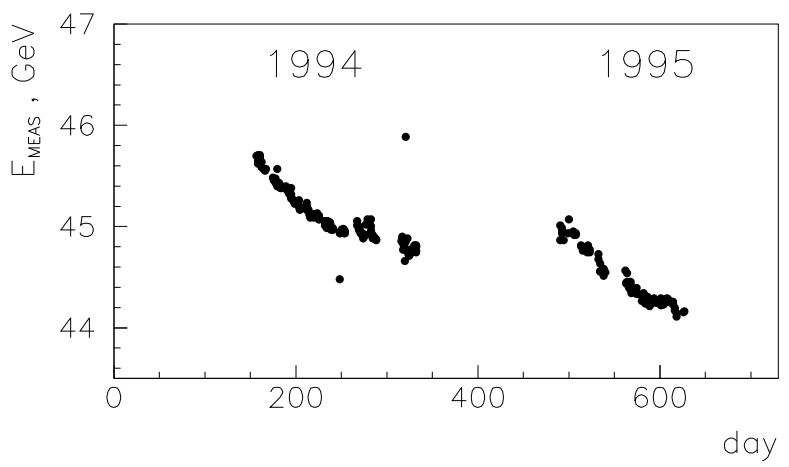

Figure 25. The calorimeter energy response to $45.6 \mathrm{GeV}$ Bhabha electrons versus time.

\section{PERFORMANCE AT LEP}

\subsection{Energy measurement}

In order to achieve a good energy resolution, a precise knowledge of the calorimeter characteristics is required. This includes a monitoring of possible changes with time. The time dependence of the energy response of the calorimeter to Bhabha electrons, obtained by using the same set of calibration coefficients for all the data, is shown in Figure 25. A reduction of the measured energy of up to $2 \%$ per year has been observed. The origin of this "ageing" effect is not well understood, but radiation damage of the scintillators and/or fibers due to the synchrotron radiation has been ruled out. However, when the fibers were changed in 1996 it was discovered that dust had accumulated on the fibers inside the sampling structure and it is possible that this could cause the observed reduction in light output.

The ageing effect is corrected for by calibrating the
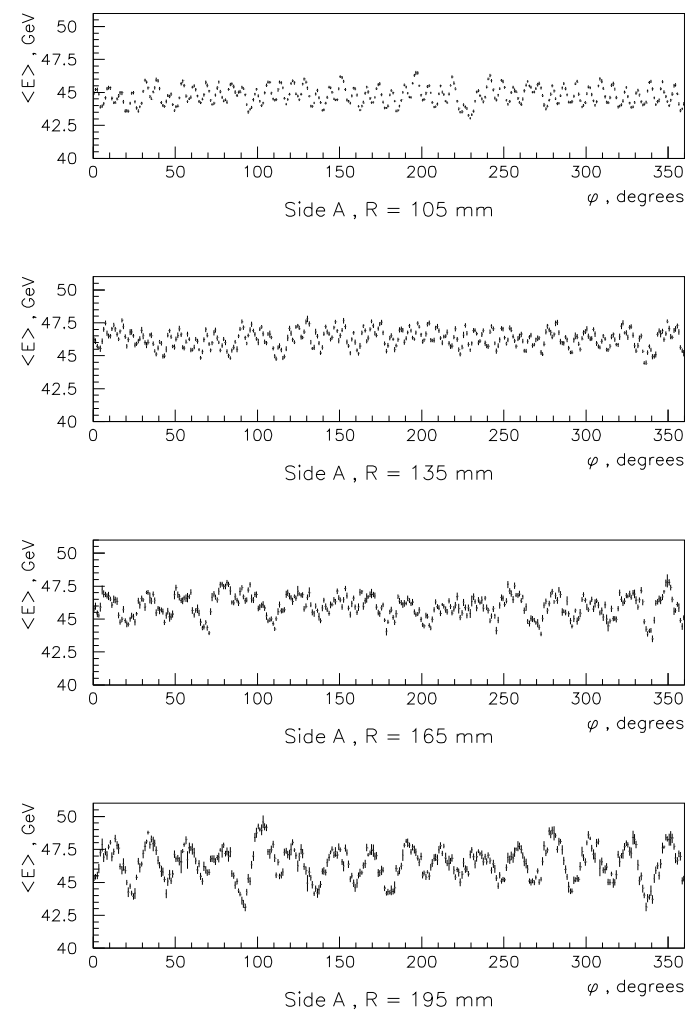

Figure 26. The energy response to $45.6 \mathrm{GeV}$ electrons as a function of the azimuthal angle at four different radii. Kuraray $\mathrm{Y} 7$ fibers were use in this measurement.

calorimeter approximately once per month, using a sample of non-radiative Bhabha events, which deposit a known amount of energy in the calorimeter. The calibration coefficients $c_{j}$ are determined from the raw amplitudes $A_{i j}$ as those minimizing [30] the quantity

$$
F=\sum_{i=1}^{N_{\text {events }}}\left(\sum_{j=1}^{N_{\text {towers }}} c_{j} A_{i j}-E_{\text {expected }}\right)^{2} .
$$

The lateral energy leakage, determined from Monte Carlo simulations, is subtracted from the beam energy to give the expected energy $E_{\text {expected }}$. The leakage is significant in the inner and the outer parts of the calorimeter, and it has been parametrized as a function of the reconstructed radial position of the shower. The steep radial distribution of Bhabha events, with many more calibration events in the inner rings than in the outer rings, was taken into account by an event-weighting procedure.

The energy response of shashlik-type calorimeters usually depends on the impact point of the incoming particle. Figure 26 shows an azimuthal scan of the energy response using Bhabha electrons and a calorimeter equipped with Kuraray Y7 fibers. In this measurement the impact point was measured by the calorimeter itself. Close to the border between tower rings one and two the fiber structure is clearly visible, and the nonuniformity of the energy response is about $\pm 3 \%$. At higher radii the fiber modulation disappears, due to the 


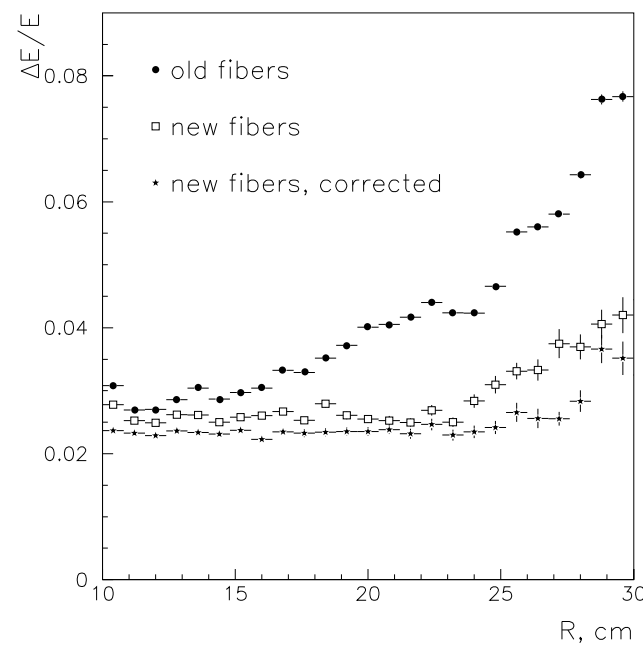

Figure 27. The energy resolution at $45.6 \mathrm{GeV}$ as a function of radius for a calorimeter with the old $\mathrm{Y} 7$ and the new $\mathrm{Y} 11$ fibers. The resolution with $\mathrm{Y} 11$ is shown both with and without the energy-map correction described in the text.

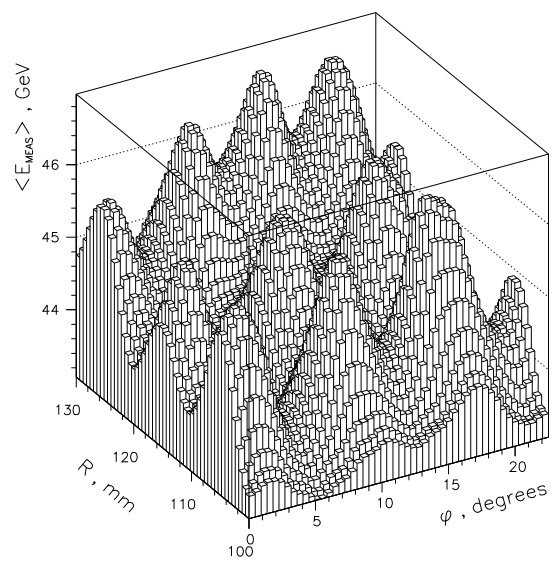

Figure 28. Energy response to $45.6 \mathrm{GeV}$ electrons as a function of the impact point (measured by the calorimeter itself).

poorer azimuthal resolution, and is replaced by a tower modulation causing non-uniformities of $\pm 6 \%$ at a radius of $19.5 \mathrm{~cm}$. This modulation is caused by the bending of the fibers at the back of the calorimeter. At high radii the towers are large in the azimuthal direction and the fibers close to the tower sector borders have to be bent more in order to be connected to the phototetrodes.

A measurement of the attenuation length as a function of the fiber bending radius showed that the Y7 fibers begin to crack when the bending radius is smaller than $8 \mathrm{~cm}$. In 1996 therefore, the Kuraray Y7 fibers were replaced with longer Y11 fibers, which are less brittle and less sensitive to bending. The improvement of the resolution due to the fiber change is demonstrated in Figure 27, which shows the energy resolution as a function of radius. At small radii where the non-uniformities are caused mainly by the increased light collection close to the fibers, there is a modest improvement of the reso-
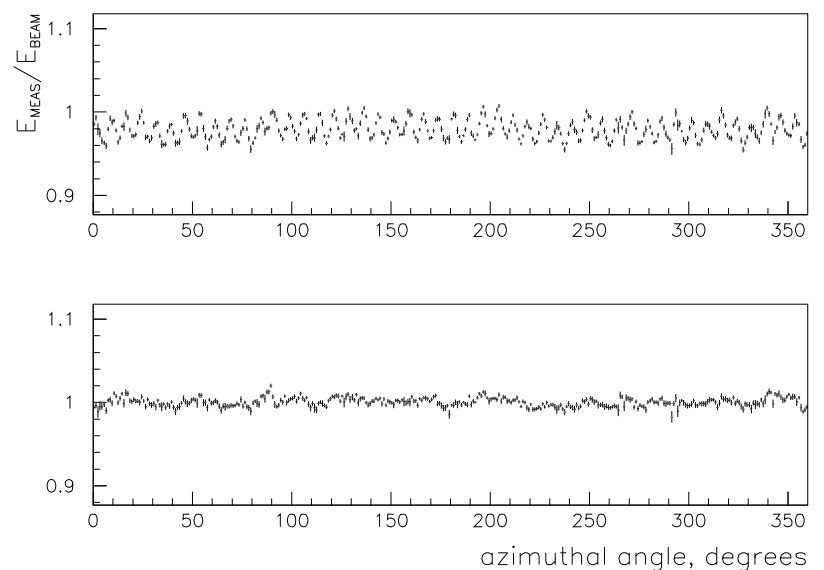

Figure 29. The energy response to $92 \mathrm{GeV}$ electrons as a function of the azimuthal angle, measured with Y11 fibers at a radius of $10.5 \mathrm{~cm}$. Both angles and radii were measured by the calorimeter itself. The upper plot shows the energies before and the lower plot those after energy-map correction.

lution. At large radii, however, where the fiber bending is the major cause of non-uniformities, a significant improvement of the energy resolution was observed with the new Y11 fibers.

The non-uniformities are reduced further by mapping the energy response as a function of the impact point. The $R-\phi$ dependence of the energy response of one STIC tower to non-radiative Bhabha events is shown in Figure 28 as a function of the reconstructed position of the shower. The peaks in the distribution correspond to the positions of WLS fibers. Figure 29 shows how the fiber modulations are reduced after applying a correction based on such an energy map. The final energy resolution at small radii after correction is given in Figure 30 as a function of the energy. The resolution predicted by GEANT simulations agrees well with the resolution measured with the new Y11 fibers. The measured resolution was parameterized as

$$
\sigma / E(\%)=(0.68 \pm 0.09) \oplus(14.1 \pm 0.4) / \sqrt{E}
$$

where the energy $E$ is in $\mathrm{GeV}$. The contribution to the second term from photoelectron statistics was estimated with the LED system to be around $5-6 \% / \sqrt{E}$ (see Section 2.4) and the resolution due to sampling fluctuations can thus be estimated to be $\sim 13 \% / \sqrt{E}$.

\subsection{Position measurement}

As mentioned previously, both the radius and the azimuthal angle of the impact point of the shower can be measured on the basis of the sharing of the deposited energy between nearby calorimeter towers. The reconstruction method relies on the testbeam measurements described in Section 6.1. A specially designed platform was used at the testbeam in order to make the beam enter the calorimeter in the same way as at LEP, i.e., following the projective geometry of the calorimeter. However, small discrepancies between the testbeam set-up 


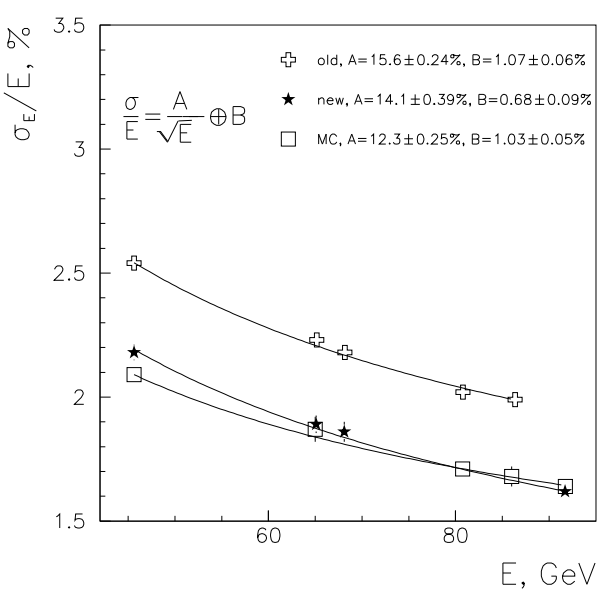

Figure 30. Energy resolution at small radii after energymap correction with the old $Y 7$ and the new $Y 11$ WLS fibers. The resolution from a GEANT Monte Carlo simulation is also shown.

and the positioning of the calorimeters at LEP together with the strongly varying resolution caused biases in the radial reconstruction. These biases were studied by comparing the measured radial distribution of Bhabha events with what was expected from the Monte Carlo simulation and a correction function was derived [31]. The size of the correction was similar to the radial resolution $(0.3-1.0 \mathrm{~mm})$.

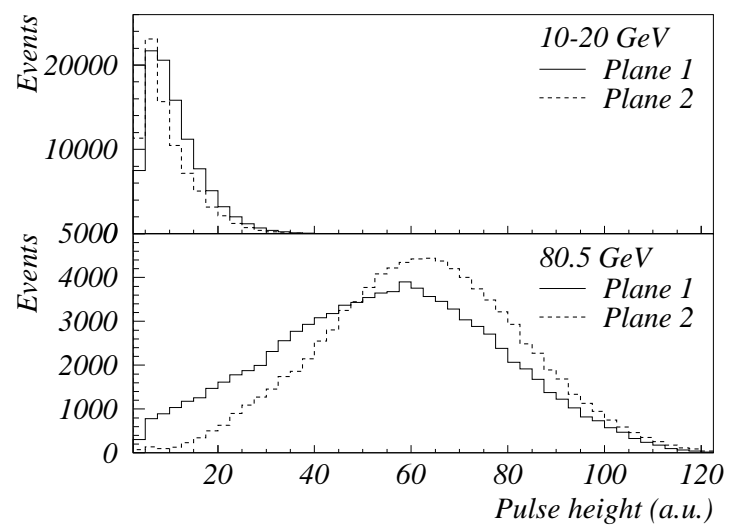

Figure 31. The pulse height distributions of the strips with the maximum signal in the silicon detectors.

The silicon strip detectors can be used to improve the measurement of the radial position and the twoshower separation. In these detectors the strips with the largest signal, after coherent noise subtraction [32], are used as the seed in the shower reconstruction algorithm [33]. The pulse height distributions of the strips with the largest signal are shown in Figure 31 for $80 \mathrm{GeV}$ Bhabha electrons and for electrons with an energy between 10 and $20 \mathrm{GeV}$.

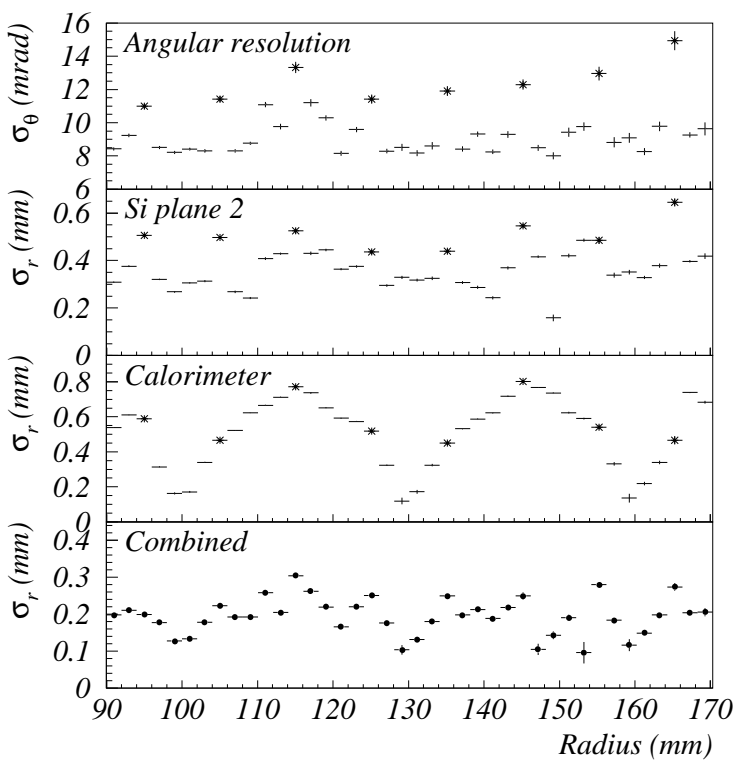

Figure 32. The angular resolution of the two silicon strip planes, the radial resolution of the second silicon strip plane, the radial resolution of the calorimeter and the radial resolution of a weighted average of the radii from the calorimeter and the two silicon planes. Points corresponding to the positions of fiber holes are marked as stars. All measurements were made with $92 \mathrm{GeV}$ electrons.

The radius and the azimuthal angle of a shower in the silicon planes are calculated by taking into account the signals of strips located at most one sector away in azimuth from the strip with the maximum and at most three strips away radially. The radial reconstruction uses the seven radial sums $\Sigma_{i}$ of pulse heights over the three sectors. After determining which sum $\Sigma_{j}$ is the largest, an estimator based on the logarithm of the fraction $\Sigma_{j-1} / \Sigma_{j+1}$ is used to calculate the radial position. The azimuthal angle is calculated with a twodimensional barycenter method, where the strips are weighted by pulse height.
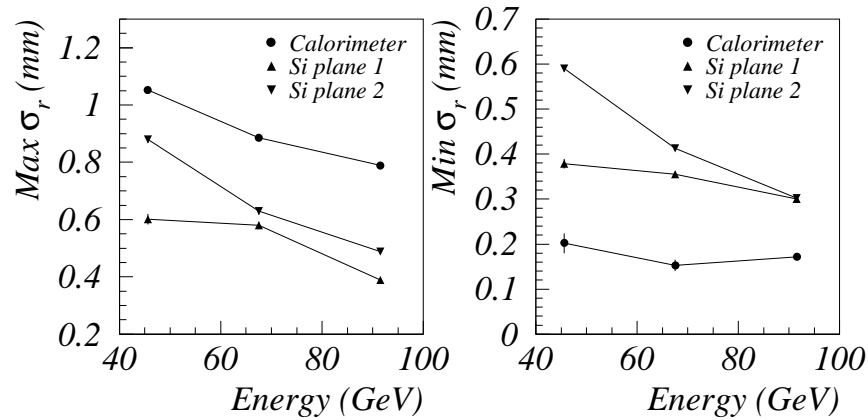

Figure 33. The radial resolution versus energy for the silicon strip planes and the calorimeter. The resolution of the silicon detector at the radii of the fiber holes is given in the leftmost plot together with the resolution of the calorimeter in the center of the towers. The rightmost plot shows the resolution in the regions between the holes in the silicon planes and at the tower borders in the calorimeter. 

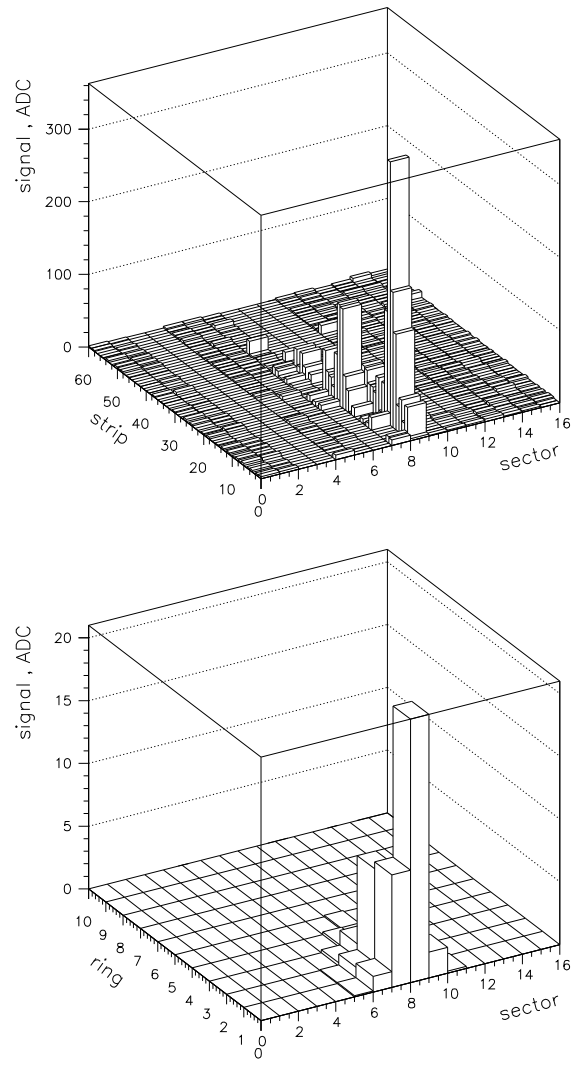

Figure 34. Two close showers are well separated in a silicon plane (top) and not separated in the calorimeter (bottom).

The uppermost plot in Figure 32 shows the angular resolution as a function of radius for $92 \mathrm{GeV}$ electrons at LEP. In this measurement, a large sample of Bhabha electrons was divided into radius bins with a width of $2 \mathrm{~mm}$, and the angular resolution was determined by fitting a Gaussian to the distribution of the differences in reconstructed radius between the two silicon planes for each bin. The resolution plot shows a regular pattern of points, separated by about $1 \mathrm{~cm}$ in radius, where the resolution is significantly poorer than elsewhere in the the detectors. These points correspond precisely to the radial locations of the holes that allow the wavelengthshifting fibers to pass through the silicon detectors. The angular resolution at $92 \mathrm{GeV}$ is approximately $9 \mathrm{mrad}$ except in the regions of the holes, where it varies between 11 and $15 \mathrm{mrad}$. At $45 \mathrm{GeV}$, the corresponding numbers are $14 \mathrm{mrad}$ and $17-25 \mathrm{mrad}$.

The three distributions of difference in radius between the two silicon planes and between each of the silicon planes and the calorimeter allowed an approximate calculation of the individual radial resolution of each detector as a function of radius. Figure 32 shows these resolutions for silicon plane 2 and for the calorimeter. While the silicon resolution is poorer in the region of the fiber holes, the calorimeter resolution shows the same behaviour as was observed in the testbeam with minima at the tower borders and maxima at the tower center. A weighted average of the three radius measurements gives a resolution of $100-300 \mu \mathrm{m}$.

The resolution of the reconstructed radii in the silicon detectors is energy-dependent and improves with increasing energy. This is shown in Figure 33 which depicts the minimum and the maximum resolution in both the silicon planes and the calorimeter as a function of energy.

In addition to the position measurement, the silicon detectors can be used to improve the two shower separation. An example of this is given in Figure 34. In this radiative Bhabha event $\left(\mathrm{e}^{+} \mathrm{e}^{-} \rightarrow \mathrm{e}^{+} \mathrm{e}^{-} \gamma\right)$, the two showers from the $\mathrm{e}^{+}$and the $\gamma$ are well separated in the silicon plane, while they are merged to one cluster in the calorimeter.
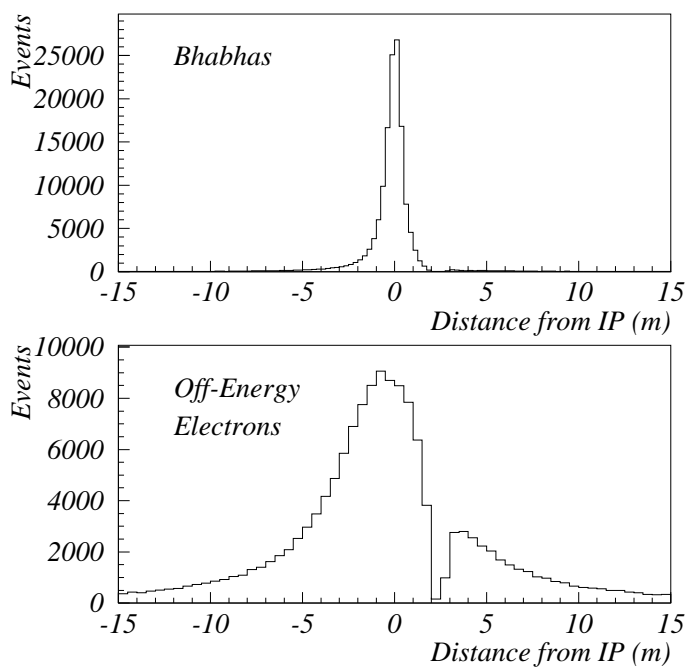

Figure 35. Distribution of reconstructed vertices of Bhabha electrons (top) and of off-energy electrons (bottom).

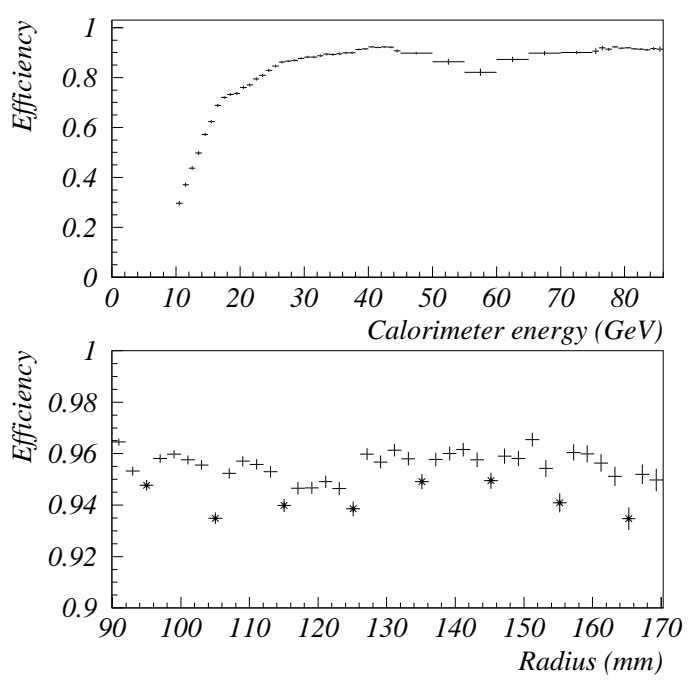

Figure 36. The efficiency of the silicon vertex reconstruction as a function of calorimeter energy (top) and radius (bottom). The points marked with stars indicate the locations of the holes for the wavelength-shifting fibers. 


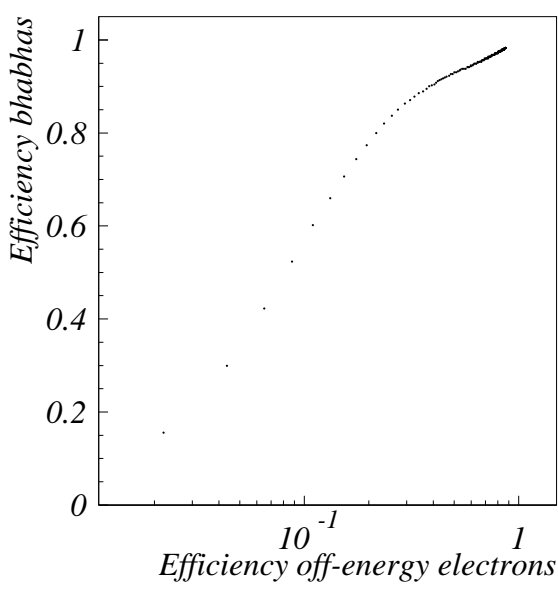

Figure 37. Fraction of Bhabha events surviving a cut on the reconstructed vertex versus fraction of off-energy electrons surviving the same cut. Each point represents an increase by $10 \mathrm{~cm}$ of the cut on the vertex, beginning with a cut at $\pm 10 \mathrm{~cm}$ near the lower left corner.

\subsection{Rejection of off-energy electrons with the silicon strip detectors}

Off-energy electrons are created in bremsstrahlung interactions between beam particles and residual gas molecules in the beampipe and may have their origin anywhere in the LEP beamline [34]. They can be rejected by a cut on the vertex reconstructed with the silicon strip detectors [33]. This vertex is calculated by extrapolating the track, formed by the two radial coordinates measured by the silicon, to the beamline. The vertex position is then defined as the distance from the intersection of the track with the beamline to the interaction point. The distributions of reconstructed vertices of a sample of $80 \mathrm{GeV}$ Bhabha electrons and of a sample of off-energy electrons are shown in Figure 35. The vertices of the Bhabha electrons are peaked in a narrow distribution around the interaction point, while the off-energy electrons have a much broader vertex distribution. The "holes" in the distributions of Figure 35, at about $2.5 \mathrm{~m}$ from the interaction point, are due to the geometrical acceptance of the silicon detectors which makes it impossible to reconstruct silicon tracks which cross the beamline close to the position of the two silicon planes themselves.

The efficiency of the vertex reconstruction is defined as the fraction of showers detected in both silicon planes. Figure 36 shows this efficiency as a function of energy. It is nearly constant at a level of $\sim 90 \%$ at energies above $25 \mathrm{GeV}$. However, the efficiency as a function of the radius is not constant since it drops by $1-1.5 \%$ at those radii where the holes for the wavelength-shifting fibers are located.

Figure 37 shows the fraction of Bhabha electrons surviving a cut on the reconstructed vertex compared to the fraction of off-energy electrons surviving the same cut. A requirement that all silicon vertices are within $\pm 0.5 \mathrm{~m}$ of the interaction point keeps $61 \%$ of the Bhabha electrons and rejects $89 \%$ of the off-energy electrons. A looser cut at $\pm 1.0 \mathrm{~m}$ keeps $80 \%$ of the electrons from the interaction point and rejects $79 \%$ of the off-energy electrons.
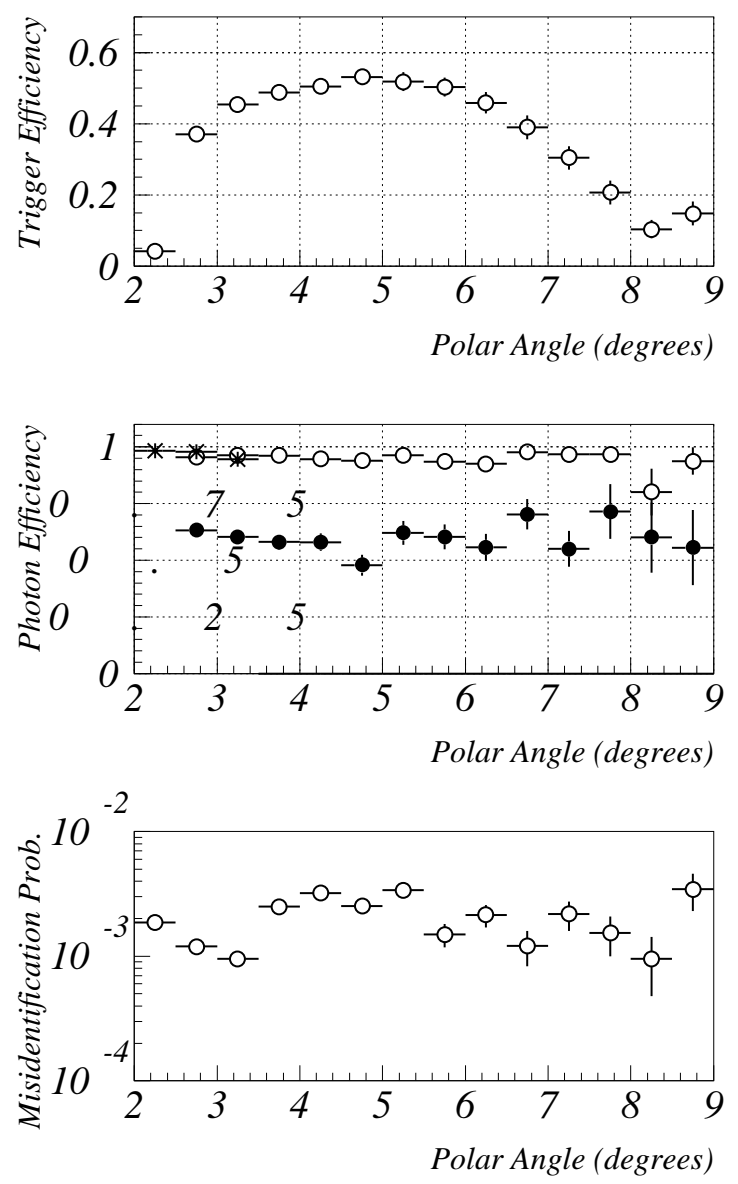

Figure 38. The trigger efficiency for photons (top), the efficiency of the offline photon identification (center) and the probability that an electron will be misidentified as a photon (bottom). In all plots open circles denote a requirement of no hits in at least one scintillator plane, filled circles no hits in either plane and stars the efficiency of the small veto counter.

\subsection{Electron-photon separation}

The efficiency of the single photon trigger has been studied with a sample of radiative Bhabha scattering events with the photon in STIC, one electron in the beampipe and the other electron in the forward lead glass calorimeter [35]. The selected photons had energies between 60 and $90 \mathrm{GeV}$. The efficiency of the single photon trigger for this sample is shown in the uppermost plot of Figure 38. The efficiency varies between $54 \%$ and $10 \%$ depending on the thickness of the material that the photons traverse before reaching the veto counter.

In the offline analysis, additional harder cuts on the veto counter pulse height were made. These had a flat efficiency of $95 \%$ (Figure 38 center). The rejection of electrons could be improved further by requiring no signal in either scintillator plane but then $35 \%$ of the pho- 


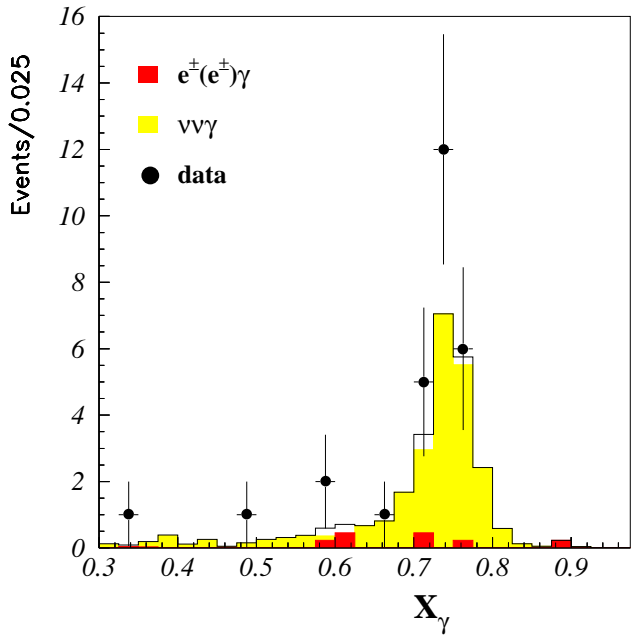

Figure 39. The ratio of the photon energy to the beam energy $\left(\mathrm{X}_{\gamma}\right)$ of single photons in STIC. The dark shaded area is the background from QED processes and the light shaded area is the expected spectrum from $\mathrm{e}^{+} \mathrm{e}^{-} \rightarrow \nu \bar{\nu} \gamma$ while the solid line is the sum of both.

tons that survived the trigger requirement and the pulse height cuts were lost (mainly due to albedo effects).

The rejection of electrons was studied with a nonradiative Bhabha sample. The probability that an electron would give a signal in one plane of the veto counter was measured to be $99.8 \%$ and the probability that an electron would be wrongly identified as a photon was measured to be $(1-4) \cdot 10^{-3}$ (Figure 38 bottom). Due to the presence of $\mathrm{e}^{+} \mathrm{e}^{-} \rightarrow \gamma \gamma$ events in the Bhabha sample, it was not possible to measure directly the rejection by the veto counter when a requirement of no signal in both planes was made. However, from the electron efficiency one can estimate it to be $\sim 4 \cdot 10^{-6}$.

Figure 39 shows the energy spectrum in STIC of single photon events at $\sqrt{s}=183 \mathrm{GeV}$ [36]. A clear radiative return peak from the process $\mathrm{e}^{+} \mathrm{e}^{-} \rightarrow \mathrm{Z}^{0} \gamma \rightarrow \nu \bar{\nu} \gamma$ is visible.

\section{THE LUMINOSITY MEASUREMENT}

At LEP the luminosity is measured by counting the number of Bhabha scattering events at low angles:

$$
\mathrm{e}^{+} \mathrm{e}^{-} \rightarrow \mathrm{e}^{+} \mathrm{e}^{-}(\mathrm{n} \gamma) \quad(\theta<200 \mathrm{mrad})
$$

The Bhabha events are selected by requiring two backto-back electromagnetic showers at small angles with respect to the beamline and having the same energy as the beams.

A calorimetric event selection is preferred since it reduces the sensitivity of the measurement to the knowledge of the material in front of the luminometer. This selection procedure is also well matched with the theoretical requirements for a precise calculation of the accepted cross section, because it retains both non- radiative and radiative Bhabhas and therefore it reduces the sensitivity to the differential distributions of any emitted photons.

The fiducial region was defined by radial cuts which were asymmetric on the two sides of the experiment (called "the narrow side" and "the wide side" respectively). This choice, common to all the experiments at LEP, minimizes the sensitivity of the measurement to movements of the interaction point and to displacements of the centers of the calorimeters with respect to the beamline [37].

In the following subsection a short summary of the key points in the luminosity determination is given. For more details see [38].

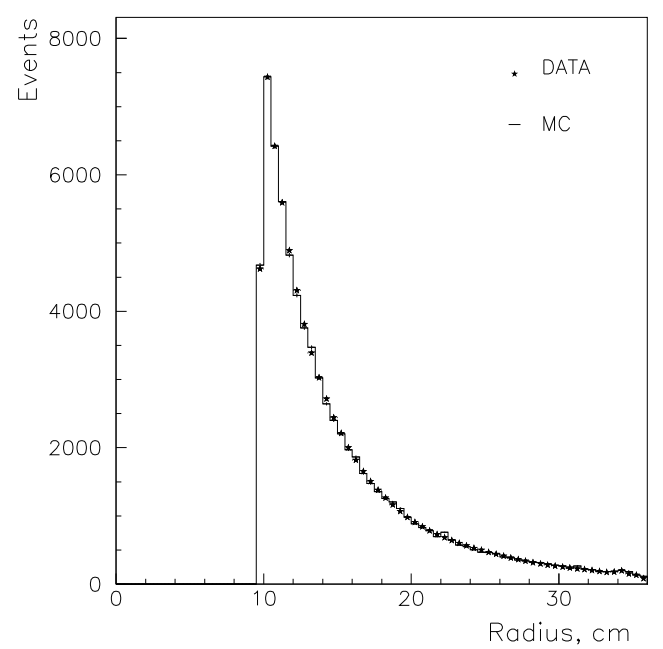

Figure 40. Radial distribution of Bhabha events measured by STIC on the narrow side and from a GEANT Monte Carlo simulation. The sharp cut at $\mathrm{R} \simeq 96 \mathrm{~mm}$ is due to the tungsten ring that defines the acceptance.

\subsection{Event selection}

The steep angular dependence of the Bhabha cross section is illustrated in Figure 40. Since the differential cross section is proportional to $1 / \theta^{3}$ the most severe requirement for the luminometer is a precise definition of the inner edge of the acceptance on the narrow side. While the other experiments at LEP1 applied a cut on the reconstructed radius of the electromagnetic showers, in DELPHI the first tower ring of the calorimeter was covered on the narrow side with a well machined tungsten ring ("the W nose"), which was $6 \mathrm{~cm}$ thick and projective to the interaction point.

As shown in Figure 41, the $\mathrm{W}$ nose made it possible to define the inner edge of the acceptance by a simple cut on the energy of the shower, since the electrons which hit the $\mathrm{W}$ nose lost most of their energy before reaching the calorimeter. 


\section{DELPHI MASK}

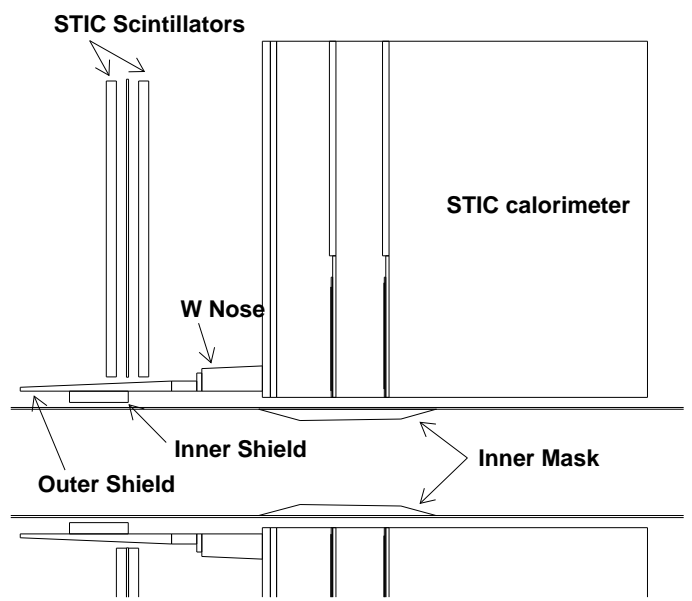

Figure 41. STIC and the tungsten shields at LEP1.

On the side opposite the mask, i.e., on the wide side, cuts were applied to the reconstructed radius of the electromagnetic shower.

The sample of Bhabha events was selected by considering, in both STIC arms, the most energetic cluster. The following selection criteria were applied:

- the energies of both clusters $\left(E_{A}\right.$ and $\left.E_{C}\right)$ had to be larger than $65 \%$ of the beam energy;

- the acoplanarity, i.e., the difference in azimuthal angle between the two clusters, should be $180^{\circ} \pm$ $20^{\circ}$;

- the reconstructed radius of the cluster on the wide side $\left(R_{A}\right)$ had to be between $8.2 \mathrm{~cm}$ and $28 \mathrm{~cm}$;

- the reconstructed radius of the cluster on the narrow side $\left(R_{C}\right)$ had to be less than $25 \mathrm{~cm}$.

The requirements on energy and acoplanarity rejected background caused by an accidental coincidence between two off-energy electrons. The energy cut also defined the inner edge of the acceptance on the narrow side, while the rest of the acceptance was determined by cuts on the reconstructed radii of the clusters.

\subsection{Evaluation of the accepted cross section}

The evaluation of the Bhabha cross section accepted in the fiducial volume of STIC was made with Monte Carlo programs in two steps. The events were generated with the BHLUMI 4.03 program [39] and they were then tracked inside the calorimeter by the GEANT program described previously (see Section 6.1).

The total theoretical uncertainty in the calculation of the accepted cross section amounted to $1.1 \cdot 10^{-3}$. It was mainly related to the truncation of the QED perturbative series, and to the technical precision in the Monte Carlo implementation.

The contribution of $\mathrm{e}^{+} \mathrm{e}^{-} \rightarrow \gamma \gamma(\gamma)$ events, which in a calorimetric measurement are indistinguishable from
Bhabha scattering events, was evaluated with another Monte Carlo program [40]. The contribution of this process was found to be $\sigma_{\gamma \gamma}=28 \mathrm{pb}$, corresponding to $5 \cdot 10^{-4}$ of the visible Bhabha cross section.
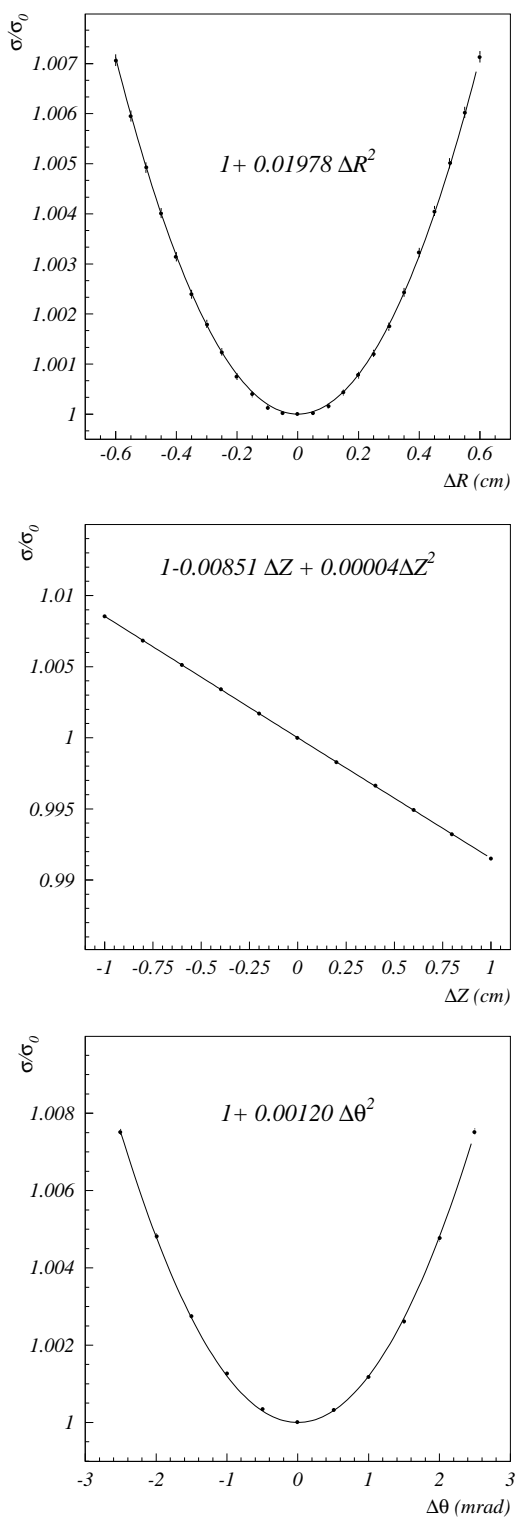

Figure 42. Dependence of the accepted cross section on the transverse position of the IP (top), on its longitudinal position (center) and on tilts of the beam (bottom).

\subsection{Effect due to IP displacements}

A displacement of the interaction point (IP) with respect to the center of the STIC system changes the cross section accepted inside the detector. This was the largest source of systematic uncertainty in the luminosity measurement.

If $D$ is the distance from the front face of the calorimeter to the interaction point, the inner edge of the acceptance becomes $R_{\text {min }}^{\prime}=R_{\min }\left(1 \pm z_{I P} / D\right)$ for a movement $z_{I P}$ in the longitudinal direction and

$$
R_{\text {min }}^{\prime}=\sqrt{\left(R_{\text {min }} \cos \phi-r_{I P}\right)^{2}+\left(R_{\min } \sin \phi\right)^{2}}
$$


for a movement $r_{I P}$ in the transverse plane along the $x$ direction.

The full $2 \pi$ coverage of the detector in azimuth cancels out the first order dependence of the visible cross section on a movement $r_{I P}$ in the transverse plane, provided that $r_{I P}$ is smaller than half the difference between the $R_{\text {min }}$ cuts on the narrow and the wide side. In this case

$$
\sigma\left(r_{I P}\right)=\sigma_{0}\left(1+2\left(\frac{r_{I P}}{R_{\min }}\right)^{2}\right)
$$

where $\sigma_{0}$ is the accepted cross section without a displacement of the IP.

The $\mathrm{W}$ nose eliminates the possibility of alternating the wide and the narrow side for each event. Therefore, in the case of a longitudinal displacements $\left(z_{I P}\right)$, the first order dependence of the visible cross section does not cancel out:

$\sigma\left(z_{I P}\right)=\sigma_{0}\left(1 \mp 2 \frac{z_{I P}}{D}+\left(\frac{z_{I P}}{D}\right)^{2}\right) \approx \sigma_{0}\left(1 \mp 2 \frac{z_{I P}}{D}\right)$.

The above relations are obtained with the assumption of a point-like luminous region and non-radiative Bhabha events. A more precise study was made with Monte Carlo simulations. Figure 42 shows that the result of the simulation of a transverse or a longitudinal displacement follows what is expected from (2) and (3). Only the coefficients in the formulae are slightly different from what is obtained with the Monte Carlo program.

Tilts of the beamline with respect to the calorimeter centerline was also studied with the simulation. As in the case of transverse displacements, it had a dependence which was quadratic in the tilt angle.

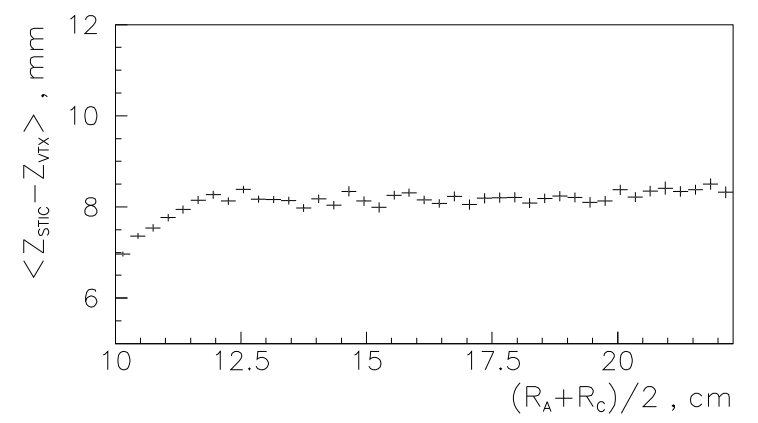

Figure 43. The difference between the positions along the beamline of the IP measured by STIC and by the microvertex detectors versus the average radius measured by the two calorimeters.

\subsection{Measurement of the IP}

The possibility of a determination of the position of the interaction point by STIC was important in order to reduce the systematic error in the luminosity measurement [31].

The position of the IP was measured by STIC, on a fill by fill basis, with a three dimensional method, by mini- mizing the quantity $\sum_{k} d_{k}^{2} / \sigma_{k}^{2}$, where $d_{k}$ is the distance from the IP to the straight line connecting the backto-back showers of the Bhabha event, corrected for the small effect of the magnetic field, while $\sigma_{k}$ is the related error.

The statistical accuracy in the reconstruction of $z_{I P}$ using Bhabha events was found to be satisfactory, and the comparison of the IP coordinates measured by STIC and by the DELPHI microvertex detector gave:

$$
\begin{aligned}
\sigma\left(x_{I P}^{S T I C}-x_{I P}^{M V T X}\right) & =22 \mu m \\
\sigma\left(y_{I P}^{S T I C}-y_{I P}^{M V T X}\right) & =61 \mu \mathrm{m} \\
\sigma\left(z_{I P}^{S T I C}-z_{I P}^{M V T X}\right) & =280 \mu \mathrm{m}
\end{aligned}
$$

As discussed above, the largest sensitivity of the accepted cross section to the IP position turned out to be in the longitudinal direction. A variation of $1 \mathrm{~mm}$ in $z_{I P}$ produced a $0.9 \%$ o change in the measured luminosity. Since $z_{I P}$ was calculated from radial measurements made by STIC, an accurate control of systematic uncertainties in the radial reconstruction over the full STIC surface was needed. In order to check this, $\mathrm{z}_{I P}$ was calculated in narrow intervals in the variable $\bar{R}=\left(R_{A}+R_{C}\right) / 2$ which is the average of the measured radii of the two showers in the Bhabha event. Figure 43 shows the dependence of $\mathrm{z}_{I P}^{S T I C}-\mathrm{z}_{I P}^{M V T X}$ on $\bar{R}$. For the region $\bar{R}>12 \mathrm{~cm}$ any bias is less than $\pm 0.3 \mathrm{~mm}$, corresponding to a systematic uncertainty of $3 \cdot 10^{-4}$ in the luminosity determination. After taking also other biasing effects of the radial reconstruction into account, a total error of $4 \cdot 10^{-4}$ was obtained. In addition, the radial mechanical positioning of the STIC towers was estimated to contribute another $3 \cdot 10^{-4}$ to the luminosity error.

Another source of systematic errors is the position of the two STIC calorimeters with respect to each other. The distance between the two calorimeters was measured to be

$$
d_{S T I C}=4400.5 \pm 0.6 \mathrm{~mm}
$$

and the $0.6 \mathrm{~mm}$ error translates into a systematic uncertainty in the luminosity of $3 \cdot 10^{-4}$. Another uncertainty of the same size stems from temperature variations in the LEP tunnel, which could alter the distance between the two calorimeters. A summary of all contributions to the systematic error from the determination of the interaction point is given in Table 2 .

\subsection{Evaluation of the systematic errors}

The major question in the luminosity analysis is how well the systematic errors in the analysis can be controlled. The estimated systematic errors from all known sources are given in Table 3 . The largest source of uncertainty, i.e., the IP position, has already been discussed. Other contributions will be described briefly below.

\subsubsection{Energy and acoplanarity cuts}

The purpose of the energy and acoplanarity cuts was to reject off-energy electron background. Figures 44 and 


\begin{tabular}{|l|c|}
\hline Source of systematics & Contribution to $\Delta \mathcal{L} / \mathcal{L}$ \\
\hline distance STIC modules & $=3 \cdot 10^{-4}$ \\
temperature effects & $=2 \cdot 10^{-4}$ \\
$\delta z_{I P}$ (mechanics) & $=3 \cdot 10^{-4}$ \\
$\delta z_{I P}$ (reconstruction) & $=4 \cdot 10^{-4}$ \\
\hline IP position & $=6 \cdot 10^{-4}$ \\
\hline
\end{tabular}

Table 2. Summary of the contributions to the systematic error due to the uncertainties of the positioning of the calorimeters with respect to the IP.

\begin{tabular}{|l|c|}
\hline Source of systematics & Contribution to $\Delta \mathcal{L} / \mathcal{L}$ \\
\hline IP position & $=6 \cdot 10^{-4}$ \\
Mask technique & $=4 \cdot 10^{-4}$ \\
MC statistics & $=3 \cdot 10^{-4}$ \\
$R_{A}^{\text {min }}$ cut & $=2 \cdot 10^{-4}$ \\
$R_{A}^{\text {max }}$ and $R_{C}^{\text {max }}$ cut & $=1 \cdot 10^{-4}$ \\
Acoplanarity cut & $=3 \cdot 10^{-4}$ \\
Energy cut & $=2 \cdot 10^{-4}$ \\
Background subtraction & $=2 \cdot 10^{-4}$ \\
Trigger inefficiency & $=0.9 \cdot 10^{-3}$ \\
\hline Total experimental & $=1.1 \cdot 10^{-3}$ \\
Total theoretical & \\
\hline
\end{tabular}

Table 3. Summary of the contributions to the systematic error on the luminosity.

45 show the variation of luminosity when the cuts are changed around their chosen values.

A variation of the energy cut between $0.6 \cdot E_{\text {beam }}$ and $0.7 \cdot E_{\text {beam }}$ translated into a variation of the luminosity of $2 \cdot 10^{-4}$. The luminosity was even less sensitive to variations of the acoplanarity cut, and the contribution to the error was estimated to be less than $1 \cdot 10^{-4}$.

\subsubsection{Geometry of the mask}

Due to the angular dependence of the Bhabha cross section, a bias $\delta R$ at the inner edge $R_{C}^{\min }$ of the acceptance on the narrow side translates into a systematic error

$$
\frac{\delta L}{L} \simeq \frac{2 \delta R_{C}^{\min }}{R_{C}^{\min }} .
$$

The goal of a $<1 \% 0$ accuracy in the luminosity determination with $R_{C}^{\min }=96 \mathrm{~mm}$ therefore leads to a requirement of $\delta R \leq 50 \mu \mathrm{m}$.

The testbeam study of the tungsten mask showed that $R_{C}^{\text {min }}$ could be measured with a resolution better than $25 \mu \mathrm{m}$. Consequently, the statistical precision $\delta R_{\text {stat }}^{\min } \simeq$ $25 \mu \mathrm{m} / \sqrt{N_{\text {Bhabhas }}}$ could, due to the large Bhabha cross section, be neglected.

In order to ensure that mechanical biases did not influence the determination of $R_{C}^{m i n}$, the mask geometry was measured in two different ways:
- a first measurement was made (with an accuracy of $3 \mu \mathrm{m}$ ) of the absolute radius in 18 azimuthal positions;

- a second measurement was made (with an accuracy of $0.1 \mu \mathrm{m}$ ) of deviations from circularity in 24 azimuthal positions.

A conservative estimation of possible biases gave $\delta R_{\text {syst }}^{\min } \simeq 20 \mu \mathrm{m}$, which translates into an error of $4 \cdot 10^{-4}$ in the luminosity determination.

\subsubsection{Radial cuts}

The radial cut at the inner border on the wide side $\left(R_{A}^{\min }=8.2 \mathrm{~cm}\right)$ cuts off the radiative tail of the Bhabha scattering distribution and it was evaluated to contribute $2 \cdot 10^{-4}$ to the systematic error. Possible biases in the radial reconstruction of up to $\sim 150 \mu \mathrm{m}$ at the outer border $\left(R_{C}^{\max }=25 \mathrm{~cm}\right.$ and $\left.R_{A}^{\max }=28 \mathrm{~cm}\right)$ gave a contribution of $2 \cdot 10^{-4}$ to the systematic uncertainty.

In addition, the mechanical accuracy of the tower positioning in the calorimeter has to be taken into account. This was measured during assembly and found to be better than $50 \mu \mathrm{m}$ in all layers (see Section 2.1). Assuming that the effective number of samplings contributing to the measurement of the spatial position of each shower is about 20, this translates into mechanical biases of about $10 \mu \mathrm{m}$ in the position determination. This is negligible compared with the biases coming from the reconstruction method itself.

\subsubsection{Energy of the beam}

The uncertainty of the measured beam energy enters into the luminosity measurement since the accepted cross section changes with energy due to two different effects:

- the QED part of the cross section has a dependence $\sigma\left(E_{\text {beam }}\right) \propto 1 / E_{\text {beam }}^{2}$;

- the $\gamma Z$ interference strongly depends on the energy (about $7 \%$ o per $\mathrm{GeV}$ ).

Close to the $\mathrm{Z}^{0}$ peak a good approximation of the uncertainty in the luminosity is given by the relation

$$
\frac{\delta L}{L} \simeq \frac{3 \delta E_{c m s}}{E_{c m s}} .
$$

Given that the uncertainty in the absolute energy of the LEP beam is at the level of $1 \mathrm{MeV}$, the contribution to the systematic uncertainty is negligible.

\subsection{Luminosity at LEP2}

During data taking at energies above the $\mathrm{Z}^{0}$ peak most physics channels of interest are statistically limited and there is no need for a very precise knowledge of the luminosity. The $\mathrm{W}$ nose used in the luminosity determination was therefore removed before the 1996 LEP run at $\sqrt{s}=161 \mathrm{GeV}$, in order to increase the forward electromagnetic coverage of DELPHI. 


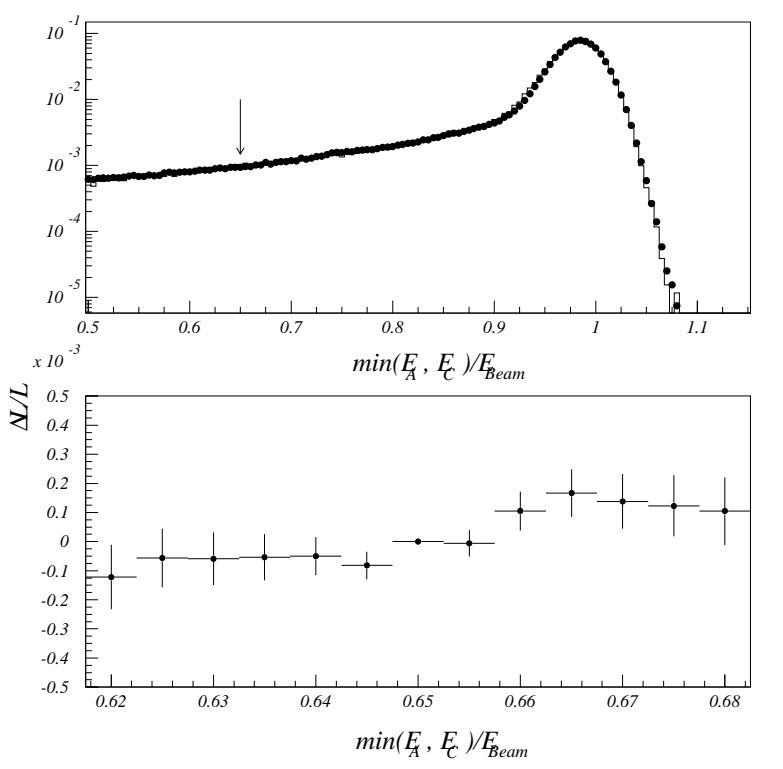

Figure 44. Comparison between data and simulation of the distribution of the energy of the lowest energy shower in Bhabha events (top). The solid line is the Monte Carlo data. The arrow indicates the cut used in the analysis. Variation of the measured luminosity versus the energy cut (bottom).

The luminosity analysis was the same as during $\mathrm{Z}^{0}$ running except that the inner acceptance on the narrow side was defined by a cut on the reconstructed radius $(R>10 \mathrm{~cm})$. After the removal of the nose, the two sides of the detector are identical, and it is possible to alternate at each event the narrow and the wide side. This reduces the sensitivity of the measurement to the position of the interaction point.

The overall systematic uncertainty is, however, completely dominated by possible biases in the reconstruction of the radius. During the high precision $\mathrm{Z}^{0}$ running in 1994, the systematic error in the Monte Carlo due to the uncertainty in the Moliere radius caused an error of $\sim 30 \mu \mathrm{m}$ in the reconstruction of the radius at a tower border. After comparing the luminosity calculated with the tungsten nose and by a radius cut $(R>13 \mathrm{~cm})$ an error of $\delta R_{s y s}^{\min } \simeq 130 \mu \mathrm{m}$ was derived for the method without the nose. This corresponds to a total uncertainty in the luminosity measurement of $2.7 \%$ o .

\section{CONCLUSIONS}

The STIC calorimeter has been operating as the DELPHI luminosity monitor since 1994 and has met the design criteria by providing a luminosity measurement with a systematic error of $0.9 \%$ at LEP 1 .

STIC is a completely hermetic detector which extends the calorimetric coverage of DELPHI both at low angle, thus improving the statistical accuracy of the luminosity measurement by a factor of two and at large angle, thus closing a gap which existed between the old luminometer and the forward electromagnetic calorimeter.
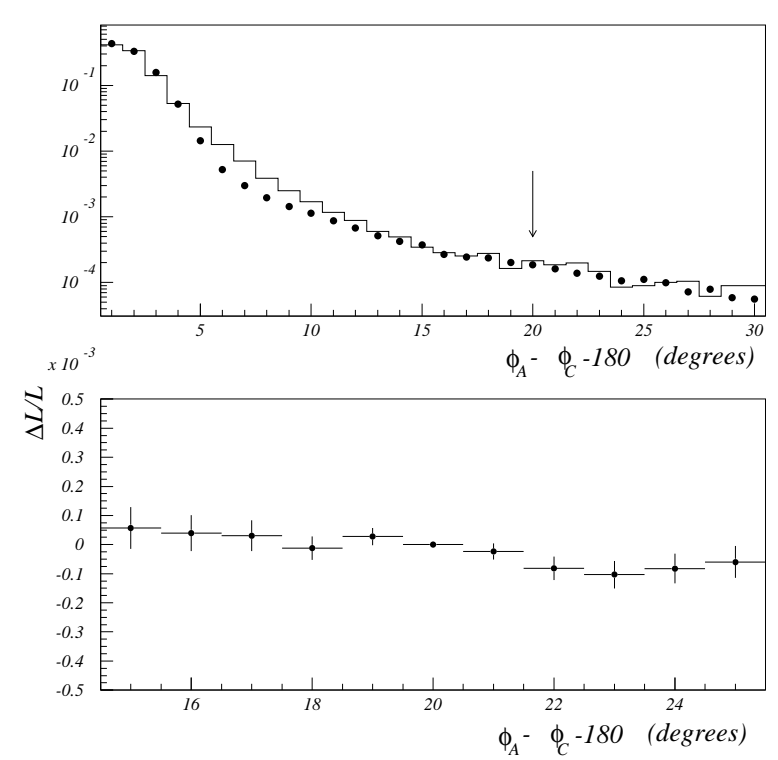

Figure 45. Comparison of the acoplanarity distribution between data and simulation (top). The solid line is the Monte Carlo data. The arrow indicates the cut used in the analysis. Variation of the measured luminosity versus the acoplanarity cut (bottom).

The calorimeter together with the silicon strip detector can measure the radial position of a shower with a resolution of 100-300 $\mu \mathrm{m}$ and the shower direction can be measured with an angular resolution of $14 \mathrm{mrad}$ at $45 \mathrm{GeV}$ and 9 mrad at $92 \mathrm{GeV}$.

The addition of the scintillator veto system allows DELPHI to measure the invisible $\mathrm{Z}^{0}$ width using the single photon trigger and it provides an effective e- $\gamma$ separation that can be used to eliminate possible backgrounds in searches for new particles.

\section{ACKNOWLEDGEMENTS}

We would like to thank the technical staff of our home institutes for their excellent work. In particular, we would like to thank M. Boldini, L. Maselli and F. Zuffa of the INFN section of Bologna, J. Pinhao, F. Ribeiro and J. Patriarca of LIP and IST, T. Nilsson of the University of Lund and P. Korobchuk, A. Zhidkikh, V. Brezhnev and L. Timshina of IHEP Serpukov. The assistance of M. Rebut and B. Buisson (CERN, EP division) was extremely valuable in the construction of the veto counters. The support of C. Brand and of the Experimental Assembly section of the Engineering and Construction group of the CERN EP division is also sincerely appreciated. We gratefully acknowledge the help we received from A. Braem (CERN, EP-TA1 section) and from C. Millerin (CERN, EP-PES section) during the fanin production. We also gladly acknowledge the support with the Kapton cable production from A. Gandi and his team at the printed circuit workshop at CERN. We would like to thank O. Runolfsson for letting us use the OPAL microvertex workshop and for his assistance during the MX4 bonding. The help from D. Regin, 
T. Taylor and R. Veness of the CERN SL division was essential during the detector installation at LEP. The assistance of the staff of the CILEA computing center in Milano during the Monte Carlo production is gratefully acknowledged.

\section{REFERENCES}

1. P. Aarnio et al., NIM A303 (1991) 233.

2. P. Abreu et al., NIM A378 (1996) 57.

3. P. Abreu et al., Nucl. Phys. B367 (1991) 511.

4. The DELPHI Collaboration, "Proposal for the replacement of the Small Angle Calorimeter of DELPHI', CERN-LEPC/92-6/P2-Add1.

5. S.J. Alvsvaag et al., Proceedings of The 5th International Conference on Calorimetry in High Energy Physics, BNL, USA, September 1994, Ed. by H.A. Gordon and D.Rueger, World Scientific 1995; S.J. Alvsvaag et al., Proceedings of The Beijing Calorimetry Symposium, Beijing, China, October 1994, Ed. by H.S. Chen, IHEP, Beijing, 1995.

6. G.S Atoyan et al., NIM A320 (1992) 144.

7. H. Fessler et al., NIM A240 (1985) 284;

B. Loehr et al., NIM A254 (1987) 26.

8. M. Bonesini et al., NIM A387 (1997) 60; M. Bonesini et al., "Study of Hamamatsu R2149 phototetrodes in magnetic field", DELPHI 96-175 CAL 133.

9. Yu. Gouz et al., "LED monitoring system for the STIC calorimeter", preprint IHEP 95-108, Protvino 1995; also available as DELPHI 95-56 CAL 126.

10. J. Janoth et al., NIM A350 (1994) 221.

11. S.J. Alvsvaag et al., IEEE Trans. Nucl. Sci. 42 (4), 478 (1995).

12. I. Lippi et al., NIM A286, (1990) 243.

13. S.J. Alvsvaag, "The SAT local trigger supervisor", Scientific/Technical report no. 1991-27, Univ. of Bergen, Norway.

14. S.J. Alvsvaag et al., "SVED The STIC veto discriminator", Scientific/Technical report no. 1996-11, Univ. of Bergen, Norway.

15. S.J. Alvsvaag et al.,"VDB The STIC veto decision box", Scientific/Technical report no. 1996-06, Univ. of Bergen, Norway.

16. T. Camporesi et al., preprint CERN-LEPC 94-13; T. Camporesi et al., preprint CERN LEPC 94-16.

17. S.J. Alvsvaag et al., IEEE Trans. Nucl. Sci. 42 (4), 469 (1995);

S.J. Alvsvaag et al., NIM A360 (1995) 219.

18. G. Della Ricca, Diploma Thesis, University of Trieste, 1993;

G. Della Ricca and M. Prest, "Simulation of the DELPHI STIC silicon shower maximum detector", preprint INFN/AE-96/04; also available as DELPHI 96-7 CAL 131.

19. S.J. Alvsvaag et al., Nucl. Phys. B (Proc. Suppl.) 44 (1995) 74;

V. Cassio, Diploma Thesis, University of Torino, 1993.

20. M. Laakso et al., NIM A326 (1993) 214.

21. J.T. Walker et al., NIM A226 (1984) 200;
P.P. Allport et al., NIM A273 (1988) 630;

J. Santon and N. Kurtz, "An introduction to the $M X$ Chip", preprint RAL-89-028.

22. M. Burns et al., NIM A277 (1989) 154.

23. A. Benvenuti et al., IEEE Trans. Nucl. Sci 40 , (1993) 537.

24. CERN Program Library Long Writeup W5013.

25. M. Bonesini et al., "Simulation of the DELPHI STIC calorimeter", DELPHI 93-118 CAL 106.

26. M. Espirito-Santo et al., "Fast simulation of an electromagnetic calorimeter: a two stage approach", DELPHI 97-42 CAL 136.

27. M. Espirito-Santo et al., "Experimental tests on scintillator tile light collection efficiency", DELPHI 97-03 CAL 134.

28. M. Paganoni et al., "Energy and position reconstruction in the DELPHI Small Angle Tile Calorimeter", DELPHI 97-07 PHYS 666.

29. A.G. Frodesen et al., "Analysis of data from the STIC prototype test", DELPHI 93-114 CAL 104.

30. J. Myrheim et al, NIM A327 (1993) 539.

31. S.J. Alvsvaag et al., IEEE Trans. Nucl. Sci. 43 (3), 1496 (1996).

32. E. Falk et al., "Algorithms for reduction of coherent noise in the silicon shower maximum detector of $S T I C^{\prime}$, preprint INFN/TC-97/33; also available as DELPHI 97-60 CAL 137.

33. E. Falk et al., "Performance of the STIC Silicon Detectors at LEP2', DELPHI 98-43 CAL 140.

34. E. Falk et al., "Simulation of Off-Energy electron background in DELPHT', preprint CERN SL/9704(EA); also available as DELPHI 97-12 LEDI 8.

35. P. Ferrari and V. Hedberg, "Photon and electron identification in the very forward region of DELPHP', DELPHI 98-49 CAL 141.

36. P. Ferrari et al., "Analysis of the single photon channel at LEPथ', DELPHI 98-55 PHYS 780;

E. Falk et al.,"Photon analysis with STIC", DELPHI 98-147 PHYS 791.

37. G. Barbiellini et al., Atti Accad. Naz. Lincei 44 (1968) 233;

J.F. Crawford et al., NIM 127 (1975) 173.

38. M. Bigi, PhD Thesis, University of Torino, 1996; $\mathrm{T}$.Camporesi et al., "Luminosity measurement in 1994 with the STIC detector", DELPHI 97-08 PHYS 667.

39. S. Jadach et al., Phys. Lett. B353 (1995) 362; S. Jadach et al., Phys. Lett. B353 (1995) 349.

40. F.A. Berends and R. Kleiss, Nucl. Phys. B186 (1981) 22. 